\title{
Facile Arene Ligand Exchange in p-Cymene Ruthenium(II) Complexes of Tertiary $P$-chiral Ferrocenyl Phosphines
}

John Popp, ${ }^{\dagger}$ Schirin Hanf, ${ }^{\dagger, \ddagger}$ Evamarie Hey-Hawkins*, ${ }^{\dagger}$

${ }^{\dagger}$ Faculty of Chemistry and Mineralogy, Institute of Inorganic Chemistry, Leipzig University, Johannisallee 29, 04103 Leipzig, Germany

${ }^{\ddagger}$ Department of Chemistry, Cambridge University, Lensfield Road, Cambridge CB2 1EW, United Kingdom

Corresponding Author: hey@uni-leipzig.de 


\section{Table of Contents}

NMR Numbering Schemes of $\mathbf{1 a}, \mathbf{b}, \mathbf{2 a}, \mathbf{b}, \mathbf{3 b}$ and $\mathbf{4 a} \mathbf{a}, \mathbf{b} \quad$ S2

X-ray Crystallography $\quad$ S4

Computational Details $\quad \mathrm{S6}$

HPLC Chromatograms $\quad$ S20

$\begin{array}{lr}\text { NMR Spectra } & \text { S24 }\end{array}$

NMR Numbering Schemes of 1a,b, 2a,b, 3b and 4a,b
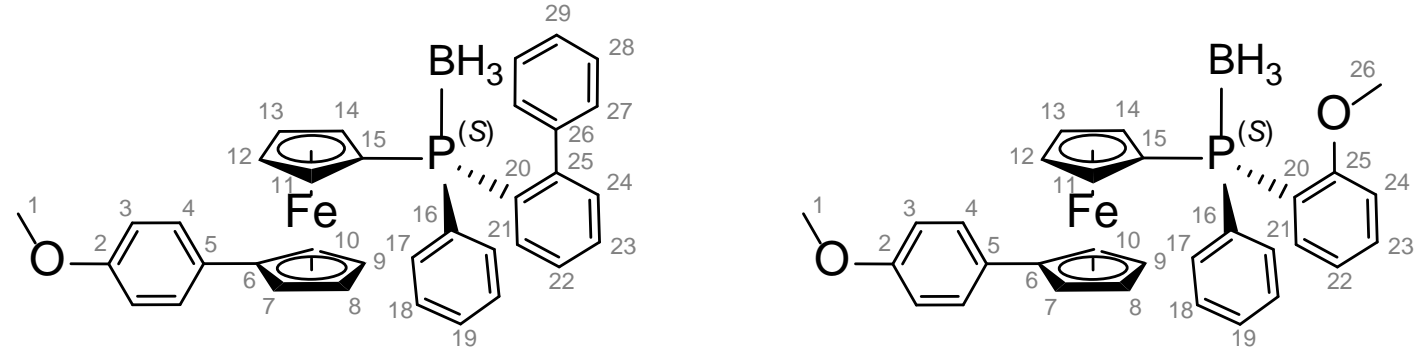

Figure S1. Numbering Scheme of 1a (left) and 1b (right).
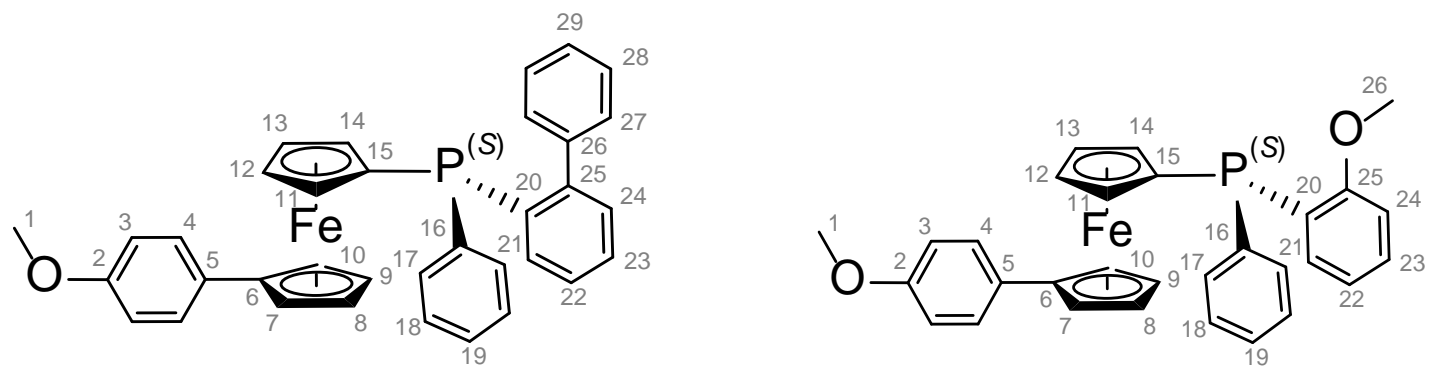

Figure S2. Numbering Scheme of 2a (left) and 2b (right). 

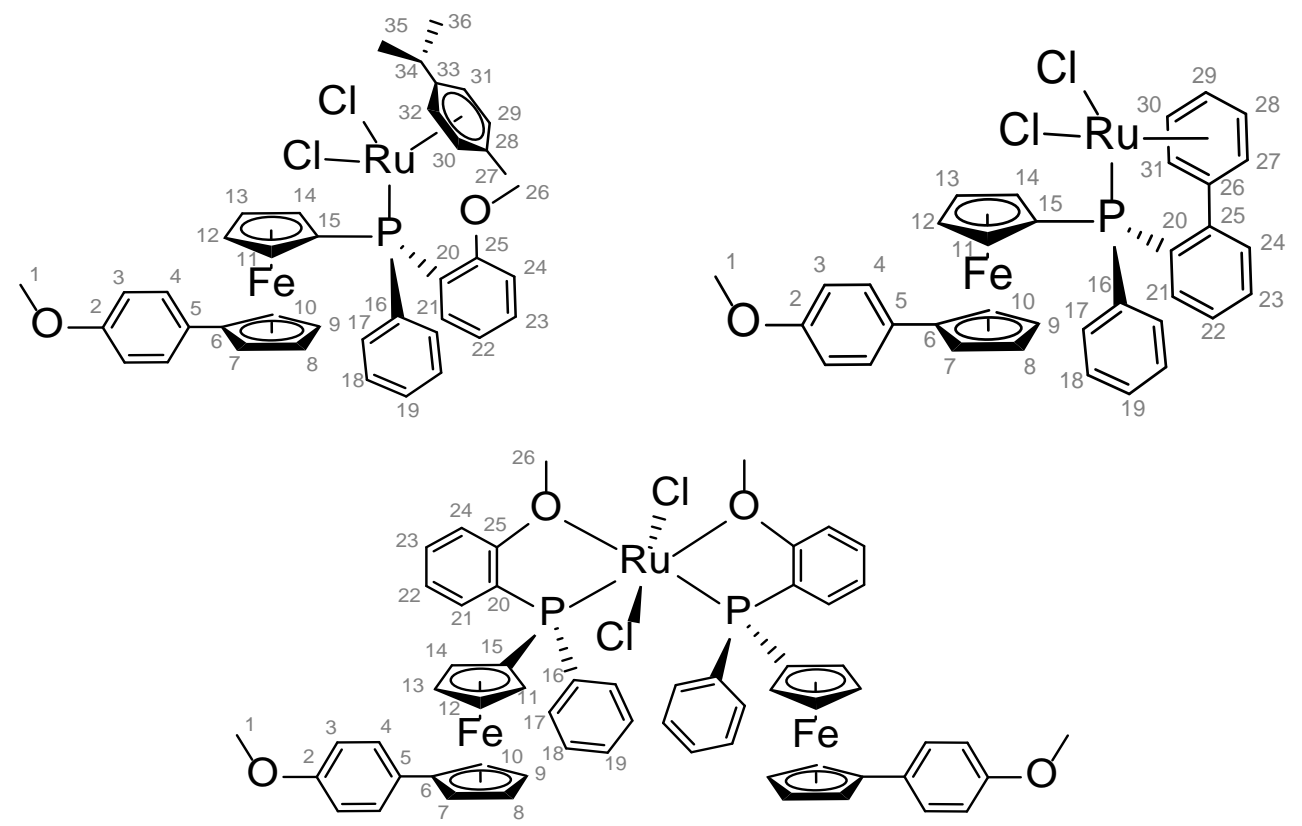

Figure S3. Numbering Scheme of $\mathbf{3 b}$ (top left), $\mathbf{4 a}$ (top right) and $\mathbf{4 b}$ (bottom). 


\section{X-ray Crystallography}

Table S1. X-ray crystallographic data of $1 \mathrm{a}, 1 \mathrm{~b}$ and $3 \mathrm{~b}$

\begin{tabular}{|c|c|c|c|}
\hline & $1 \mathbf{a}$ & $\mathbf{1 b}$ & $\mathbf{3 b} \cdot \mathrm{CH}_{2} \mathrm{Cl}_{2} \cdot 0.25 \mathrm{C}_{6} \mathrm{H}_{12}{ }^{a}$ \\
\hline empirical formula & $\mathrm{C}_{35} \mathrm{H}_{32} \mathrm{BFeOP}$ & $\mathrm{C}_{30} \mathrm{H}_{30} \mathrm{BFeO}_{2} \mathrm{P}$ & $\mathrm{C}_{42.5} \mathrm{H}_{46} \mathrm{Cl}_{4} \mathrm{FeO}_{2} \mathrm{PRu}$ \\
\hline formula weight $[\mathrm{g} / \mathrm{mol}]$ & 566.23 & 520.17 & 918.48 \\
\hline $\mathrm{T}[\mathrm{K}]$ & 130 & 130 & 130 \\
\hline crystal system & orthorhombic & orthorhombic & triclinic \\
\hline space group & $P 2_{1} 2_{1} 2_{1}$ & $P 2{ }_{1} 2_{1} 2_{1}$ & $P \overline{1}$ \\
\hline$a[\AA]$ & $7.2519(3)$ & $8.0956(2)$ & $10.0321(2)$ \\
\hline$b[\AA]$ & $16.6879(9)$ & $10.9588(3)$ & 12.8901(3) \\
\hline$c[\AA]$ & 23.0206(9) & $28.2204(7)$ & 17.3984(3) \\
\hline$\alpha\left[^{\circ}\right]$ & 90 & 90 & $90.327(2)$ \\
\hline$\beta\left[^{\circ}\right]$ & 90 & 90 & $101.504(2)$ \\
\hline$\gamma\left[^{\circ}\right]$ & 90 & 90 & $110.961(2)$ \\
\hline$V\left[\AA^{3}\right]$ & $2785.9(2)$ & 2503.66(11) & 2051.46(8) \\
\hline$Z$ & 4 & 4 & 2 \\
\hline$\rho_{\text {calcd }}\left[\mathrm{g} \cdot \mathrm{cm}^{-3}\right]$ & 1.350 & 1.380 & 1.487 \\
\hline$\theta_{\max }\left[^{\circ}\right]$ & 27.989 & 26.367 & 30.507 \\
\hline$F(000)$ & 1184 & 1088 & 940 \\
\hline reflns collected & 20902 & 20242 & 67517 \\
\hline independent reflns & 5898 & 5103 & 12522 \\
\hline$R 1 / w R 2[\mathrm{I}>2 \sigma(\mathrm{I})]$ & $0.0661 / 0.1078$ & $0.0334 / 0.0605$ & $0.0386 / 0.1066$ \\
\hline$R 1 / w R 2$ (all data) & $0.1312 / 0.1307$ & $0.0411 / 0.0632$ & $0.0459 / 0.1107$ \\
\hline Flack parameter $x$ & $0.00(2)$ & $0.005(8)$ & - \\
\hline Largest diff. peak/hole $\left[\mathrm{e} \cdot \AA^{-3}\right]$ & $0.482 /-0.790$ & $0.221 /-0.213$ & $2.586 /-1.097$ \\
\hline
\end{tabular}

${ }^{a}$ The solid-state structure of $\mathbf{3 b}$ contains one $\mathrm{CH}_{2} \mathrm{Cl}_{2}$ molecule and formally a quarter of a cyclohexane molecule per asymmetric unit. The disordered cyclohexane (isotropic refinement) is located on a special position (inversion center). 
Table S2. X-ray crystallographic data of $4 a$ and $4 b$

\begin{tabular}{|c|c|c|}
\hline & $\mathbf{4 a} \cdot 2 \mathrm{CH}_{2} \mathrm{Cl}_{2}{ }^{b}$ & $4 \mathbf{b} \cdot \mathrm{CHCl}_{3}{ }^{c}$ \\
\hline empirical formula & $\mathrm{C}_{37} \mathrm{H}_{33} \mathrm{Cl}_{6} \mathrm{FeOPRu}$ & $\mathrm{C}_{61} \mathrm{H}_{55} \mathrm{Cl}_{5} \mathrm{Fe}_{2} \mathrm{O}_{4} \mathrm{P}_{2} \mathrm{Ru}$ \\
\hline formula weight $[\mathrm{g} / \mathrm{mol}]$ & 894.22 & 1304.19 \\
\hline $\mathrm{T}[\mathrm{K}]$ & 130 & 130 \\
\hline crystal system & triclinic & orthorhombic \\
\hline space group & $P \overline{1}$ & $P 2_{1} 2_{1} 2_{1}$ \\
\hline$a[\AA]$ & 8.7032(8) & $10.8773(2)$ \\
\hline$b[\AA]$ & $14.382(2)$ & 19.3811(4) \\
\hline$c[\AA]$ & $15.747(2)$ & $52.879(1)$ \\
\hline$\alpha\left[^{\circ}\right]$ & $70.00(1)$ & 90 \\
\hline$\beta\left[^{\circ}\right]$ & $86.67(1)$ & 90 \\
\hline$\gamma\left[^{\circ}\right]$ & 73.762(9) & 90 \\
\hline$V\left[\AA^{3}\right]$ & $1776.7(4)$ & $11147.6(4)$ \\
\hline$Z$ & 2 & 8 \\
\hline$\rho_{\text {calcd }}\left[\mathrm{g} \cdot \mathrm{cm}^{-3}\right]$ & 1.672 & 1.554 \\
\hline$\theta_{\max }\left[^{\circ}\right]$ & 26.560 & 26.797 \\
\hline$F(000)$ & 900 & 5313 \\
\hline reflns collected & 11416 & 59414 \\
\hline independent reflns & 5930 & 20708 \\
\hline$R 1 / w R 2[\mathrm{I}>2 \sigma(\mathrm{I})]$ & $0.0754 / 0.0909$ & $0.0779 / 0.1793$ \\
\hline$R 1 / w R 2$ (all data) & $0.1844 / 0.1183$ & 0.0928/0.1892 \\
\hline Flack parameter $x$ & - & $-0.007(10)$ \\
\hline Largest diff. peak/hole $\left[\mathrm{e} \cdot \AA^{-3}\right]$ & $0.781 /-0.682$ & $2.299 /-3.587$ \\
\hline
\end{tabular}

${ }^{b}$ The solid-state structure of $\mathbf{4 a}$ contains two $\mathrm{CH}_{2} \mathrm{Cl}_{2}$ molecules per asymmetric unit. ${ }^{c}$ The solid-state structure of $\mathbf{4 b}$ contains two $\mathrm{CHCl}_{3}$ molecules per asymmetric unit (one anisotropically refined, the other heavily disordered with isotropic refinement). 


\section{Computational Details}

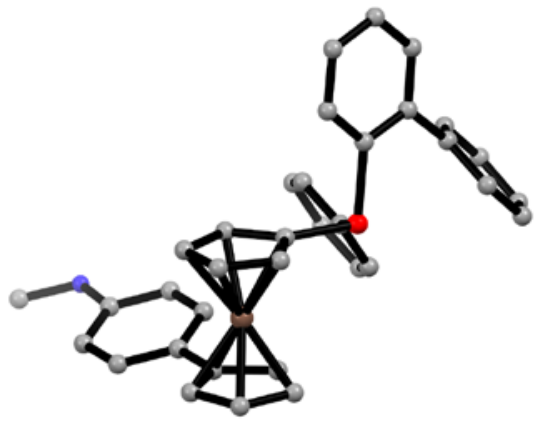

$2 a$

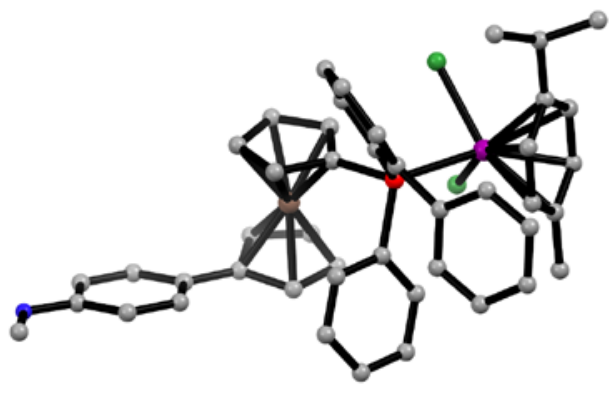

$3 a$

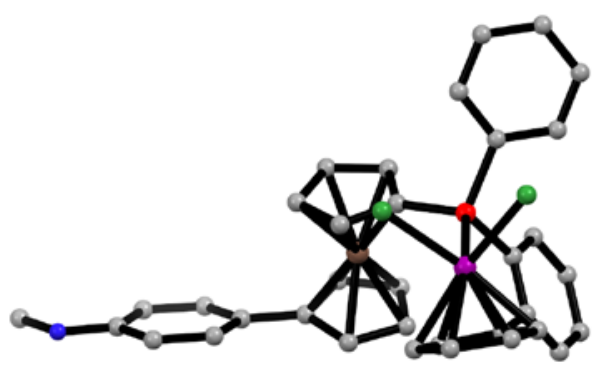

$4 a$

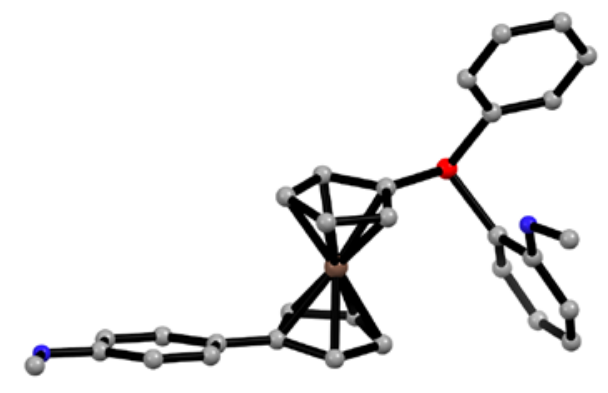

2b

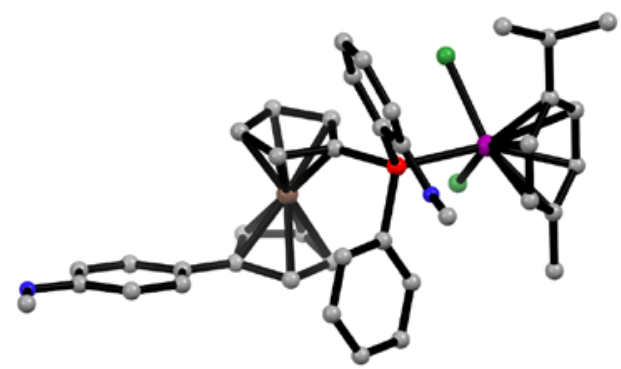

$3 b$

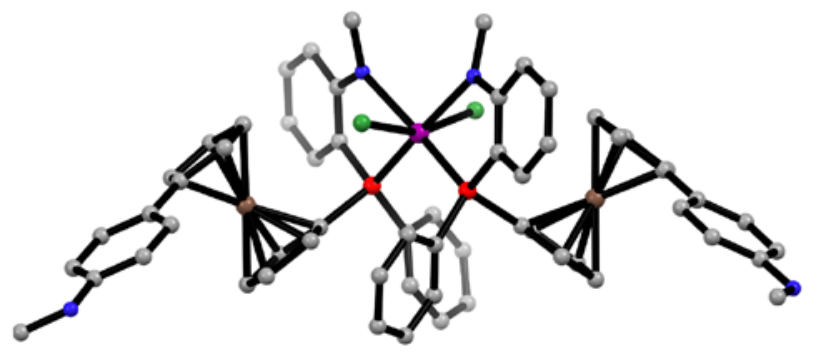

4b

Figure S4. Geometrically optimized structures of $\mathbf{2 a , b}, \mathbf{3} \mathbf{3}, \mathbf{b}$ and $\mathbf{4 a , b}$. 


\begin{tabular}{|c|c|c|c|}
\hline \\
\hline \multicolumn{4}{|c|}{$E=-3032.02277588 E h$} \\
\hline & 5.92937842408816 & 15.97082574449240 & 4.50836323983670 \\
\hline & 5.44330228184551 & 15.87946882644264 & 3.19724824418028 \\
\hline & 5.99531467547804 & 16.74683103868038 & 15455 \\
\hline & 6.987 & & \\
\hline & 7.46688841594472 & 17.7 & 559 \\
\hline & 6.930956 & 16.8807 & 21651 \\
\hline & 4.4056 & 14.90 & 0606 \\
\hline & 3.59165 & 14.9 & \\
\hline & 2.736 & 24 & \\
\hline & 3.01 & & \\
\hline & 4.0389 & 13.7 & 770 \\
\hline & 4.702 & 13.2 & \\
\hline & 6.58297 & 2219 & 436 \\
\hline & 5.636 & 12 & 978 \\
\hline & 5.01 & & \\
\hline & 5.582 & & \\
\hline & 6.545 & & \\
\hline & 5.14519434639735 & 13.80 & -1.6 \\
\hline & 8.449 & 18.6 & \\
\hline & 8.958 & & \\
\hline & 5.6960 & 15.5 & $-1.3^{3}+x-5$ \\
\hline & 7.0432 & & \\
\hline & 7.4079 & 17.2 & -1.0 \\
\hline & $6.4311 t$ & 18.2 & -1.0 \\
\hline & 5.088 & 90 & -1 \\
\hline & 4.725 & & -1 \\
\hline & 6.50835 & & -2.7 \\
\hline & 6.7016 & 13.9 & 5495 \\
\hline & 7.747 & & -4 \\
\hline & 8.578 & 12. & -4 \\
\hline & $8.3710 €$ & $11.7 \varepsilon$ & -3.2 \\
\hline & 7.34811 & 12.18 & -2.42706942040579 \\
\hline & 5.8400 & 15.0 & -4.37 \\
\hline & 4.4563 & 2475 & -4.5 \\
\hline & 3.645 & 2665 & -4.85325094627870 \\
\hline & 4.20390501 & 17.28058625 & -4.98799921925631 \\
\hline & 5.58241098007542 & 17.45094295043204 & -4.83604868556180 \\
\hline & 12271370 & 16.35606829935748 & -4.54006879478173 \\
\hline & 4.24191781137475 & 11.22075928208422 & -0.21714377308241 \\
\hline
\end{tabular}



H 5.30267994649890
10.48488250385751
2.17128701999343
H 7.11802914084490
12.36884173487343
2.88897864353197
H 7.17480785476188
14.25915720862491
0.96193006862229
H 3.63530456765149
15.67225937166606
0.86343813160295
H 2.02762386418071
13.50078427654215
0.88601080527643
H 2.55027238904453
12.09763128021123
3.15188825967037
H 4.49664553772531
13.39328294556393
4.50273252379887
H 7.81182562712874
15.13141301806244
$-1.20395306235945$
H 8.45861960037896
17.51017036302469
$-0.92427645675174$
H 6.71755940092783
19.29266428670819
$-0.98023837454179$
H 4.32359485832391
18.67575901359038
$-1.28907537141334$
H 3.68169435230057
16.28628012323487
$-1.55414388799673$
H 7.89415635884237
14.06139702383582
$-5.77299038375015$
H 9.38052986058756
12.15679358707254
$-5.16477674589316$
H 9.01390926844072
10.94605404231202
$-3.00983803662226$
H 7.21246976734614
11.67465843366068
$-1.47365857241506$
H 4.01850937520575
13.92104537855734
$-4.43629341283590$
H 2.57113107890777
15.86431065332863
$-4.98281933495266$
H 3.56731770889062
18.13762338061014
$-5.21220766842372$
H 6.02584147969459
18.44270568701653
$-4.93375614761183$
H 7.46560854268122
16.49246225454961
$-4.38834043343888$
H 5.65675165541658
16.69198717384686
1.19886274985475
18.31728621715299
1.81119368294052
16.92055028203744
5.89127887324311
H 7.27682037708148
15.32811268863305
5.28381595708600
H 5.51044179398836
19.04161102679713
6.15397577647901
H 9.72444460128026
19.53785440377160
5.41083327555263
H 9.41552572413337
17.80601283773582
5.76647656889757

\section{3a}

$E=-4437.25116286 \mathrm{Eh}$

C 7.76755676572628

C 8.02526454000878

C 9.35888624467298

C 9.90317036533716

C 8.92469079246953

P 10.31167154453630

C 11.80961448151228

C 13.11686012547253

C 14.08309439871807
2.73480246469843

1.64880509100434

1.16737560541488

1.96669465545321

2.93497175524940

$-0.08092578319465$

0.89427455174107

0.41927918737155

1.37172854474634
4.97551692129680

4.08551400640100

4.33729580657018

5.41788106088617

5.79573436460377

3.40936210450445

2.90288684950966

2.58693845176238

2.19912719449776 


\begin{tabular}{|c|c|c|c|}
\hline & & & \\
\hline & 256278 & & \\
\hline & & & \\
\hline & & & \\
\hline & & & \\
\hline & 93 & 59 & 14 \\
\hline & & & \\
\hline & & -0.4 & \\
\hline & & & \\
\hline & & 755 & 919 \\
\hline & & & \\
\hline & & & 927 \\
\hline & & & \\
\hline & & & \\
\hline & & & \\
\hline & & & \\
\hline & & & \\
\hline & & & 955 \\
\hline & & & \\
\hline & & & \\
\hline & & -1.8 & -0 \\
\hline & & & \\
\hline & & -3.3 & \\
\hline & & & 211 \\
\hline & & & \\
\hline 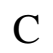 & & -44 & \\
\hline & & & \\
\hline & & & \\
\hline & & & \\
\hline $\mathrm{C}$ & 96 & 04 & \\
\hline $\mathrm{C}$ & & & \\
\hline & 18 & & 655 \\
\hline & 61 & 239 & 820 \\
\hline & & & 510 \\
\hline & & & 21 \\
\hline & & & \\
\hline 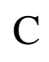 & 443 & & 7680 \\
\hline $\mathrm{H}$ & 7459659 & -1.47262527987670 & 74825557 \\
\hline & .15 & & 7.808 \\
\hline & & & \\
\hline & & & \\
\hline
\end{tabular}




\begin{tabular}{|c|c|c|c|}
\hline & & & \\
\hline & 283752983498 & 0.52723003474335 & -1.4 \\
\hline & & & \\
\hline & & & \\
\hline & & & \\
\hline & & & 747 \\
\hline & 73 & 1.22 & 3.35 \\
\hline & & -2.7 & \\
\hline & & & \\
\hline & & & \\
\hline & & & \\
\hline & & & 11.4 \\
\hline & & & 66 \\
\hline & & & 325 \\
\hline & & & \\
\hline & & & \\
\hline & & -1.2 & -3 \\
\hline & & -1 . & \\
\hline & & & \\
\hline & & & \\
\hline & & & \\
\hline & & 3 & \\
\hline & & & \\
\hline & 1 & -5.3 & 1.7 \\
\hline & & -4.2 & \\
\hline & & -( & \\
\hline & & & \\
\hline & & & \\
\hline & & & \\
\hline & & & \\
\hline & & & \\
\hline & & & \\
\hline & & 479 & 1036 \\
\hline & & 3.27 & 5.05 \\
\hline & & & \\
\hline & & & \\
\hline & & & \\
\hline 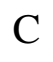 & & -3 & 92547 \\
\hline & 341 & 2019 & 472854765 \\
\hline & & & \\
\hline & & & \\
\hline
\end{tabular}




$\begin{array}{llll}\text { H } & 15.00134033757205 & -4.61789128840793 & 1.88935607100806 \\ \text { H } & 14.10553601797466 & -3.94818643369356 & 4.12555263079941 \\ \text { H } & 13.25809459750701 & -1.68709984036982 & 4.49720927358137\end{array}$

4a

$E=-4047.75346314 \mathrm{Eh}$

$\mathrm{Ru} 3.72986064280220$

6.61265917781658

2.30348446424902

Fe 4.51102436154680

6.38408666330821

7.17942931864085

Cl 4.45200143544597

5.07167285646063

0.63919713377648

Cl 2.01651356967526

5.04392608612483

2.87293297792866

P 5.11100759906070

5.57838318398915

3.80075028034499

O -0.80513838875341

7.33751968056550

11.69764474852269

C 4.47034170769470

5.25971320238094

5.45852257625020

C 5.17059972295160

4.56939701692595

6.51382850497136

H 6.19727238930779

4.21945738480404

6.47132626384019

C 4.26303267534753

4.40024530642103

7.60703091006550

H 4.49460893919556

3.93565326775102

8.56119324395712

C 3.01081547483858

4.99123193989505

7.24102456391846

H 2.13539254081795

5.07153434034938

7.87946730789242

C 3.13425646904507

5.52685204867753

5.92381999676275

H 2.35858173214739

5.99987321087663

5.33189894225193

C 6.46660838460076

6.79591566014041

4.02848687395334

C 7.65918459597332

6.53953655179392

4.71350629589122

H 7.85421227982317

5.54267829927262

5.10935593956281

C 8.60129867661672

7.55645291897605

4.87595702312515

H 9.53257277412560

7.35420933754051

5.40641771527513

C 8.35790554363750

8.83088756374657

4.35326146320082

H 9.09706151290948

9.62254590285893

4.47948581946419

C 7.17057365697632

9.09287257326703

3.66483344504393

H 6.97536533853700

10.08568278451203

3.25676201798849

C 6.22199671044093

8.07828158435767

3.50021368202212

C 4.92342345992218

8.29933275204569

2.80066171807334

C 4.84409210532156

8.23407381654739

1.37625458425508

H 5.75148889734182

8.08603036341893

0.79429641758586

C 3.56268905053793

8.23465773521433

0.72858470799141

H 3.51968563600991

8.07790843475156

$-0.34855798008872$

C 2.37750430756021

8.26592082138381

1.47791959825935

H 1.41202699980114

8.14699096688734

0.99059638071108

C 2.45849506266351

8.29061850139474

2.91499217371542

H 1.54697636888424

8.19913305430903

3.50440323175391 


$\begin{array}{clcc}\text { C } & 3.70562169057171 & 8.39328177577619 & 3.57651585523947 \\ \text { H } & 3.75748399601387 & 8.39214759028410 & 4.66377862010226 \\ \text { C } & 5.90627376714443 & 4.01337854427697 & 3.35905989992572 \\ \text { C } & 7.08555011931927 & 3.98993038277405 & 2.60251047375048 \\ \text { H } & 7.57217039793885 & 4.92433628748708 & 2.32298726412585 \\ \text { C } & 7.62538857257513 & 2.77059660839610 & 2.19429027098754 \\ \text { H } & 8.54470370482486 & 2.75532390099104 & 1.60753770807849 \\ \text { C } & 6.97933370012597 & 1.57480024531087 & 2.51899285971025 \\ \text { H } & 7.39855916368909 & 0.62247068294525 & 2.19135285208076 \\ \text { C } & 5.78810838445016 & 1.59924136391645 & 3.24982354775192 \\ \text { H } & 5.27205032691166 & 0.66839594735065 & 3.48790057338821 \\ \text { C } & 5.24875759437353 & 2.81440996708877 & 3.67003412976317 \\ \text { H } & 4.30828903467262 & 2.83927969312989 & 4.22083061712381 \\ \text { C } & 3.94132058035516 & 7.70776550416074 & 8.64052535606924 \\ \text { C } & 4.07172121229035 & 8.37598028894946 & 7.36924249217184 \\ \text { H } & 3.28320544529810 & 8.93059408494878 & 6.86816497214009 \\ \text { C } & 5.41514747427699 & 8.21644764971761 & 6.90668547928680 \\ \text { H } & 5.82590924277260 & 8.60911507564247 & 5.98299871908724 \\ \text { C } & 6.12939620525500 & 7.43948606318158 & 7.87158526029652 \\ \text { H } & 7.16656290322958 & 7.12871814823090 & 7.79459088271713 \\ \text { C } & 5.22684673123723 & 7.12568520650923 & 8.93741060146534 \\ \text { H } & 5.45695937706626 & 6.51221891403510 & 9.80353561121014 \\ \text { C } & 2.72730342167826 & 7.61369882344392 & 9.45670823956998 \\ \text { C } & 1.45023532020375 & 7.80263834677476 & 8.89006550094329 \\ \text { H } & 1.36294500650981 & 8.00172068771036 & 7.82125892370915 \\ \text { C } & 0.29836040359965 & 7.70655858287326 & 9.65563586820095 \\ \text { H } & -0.68772211851214 & 7.84544384212039 & 9.21181586201255 \\ \text { C } & 0.38451454165844 & 7.41430812755825 & 11.02789899314411 \\ \text { C } & 1.64296714749193 & 7.22406168270911 & 11.61288219492961 \\ \text { H } & 1.74061048915928 & 7.00331671757365 & 12.67430129803245 \\ \text { C } & 2.79385674901166 & 7.32706932357850 & 10.82643636069096 \\ \text { H } & 3.76734564108914 & 7.19508108370663 & 11.30038577595410 \\ \text { C } & -0.75512262409159 & 7.03565584288875 & 13.09044461360325 \\ \text { H } & -0.29273072939901 & 6.05122747491110 & 13.27178291238273 \\ \text { H } & -1.79621473290901 & 7.01773397414864 & 13.43017019635866 \\ \text { H } & -0.19981137268955 & 7.80730857613435 & 13.64892787283934\end{array}$




\begin{tabular}{|c|c|c|c|}
\hline \\
\hline \\
\hline $\mathrm{C}$ & 1.20560465034092 & 10.50222077384511 & 23.592300 \\
\hline & & & 577 \\
\hline C & & & \\
\hline $\mathrm{C}$ & -0.88260589504647 & 12.34489353338516 & 20651 \\
\hline C & 0573666761 & 11.39980790949707 & 21836 \\
\hline C & 0.044 & 78000915355 & 22.78 \\
\hline $\mathrm{O}$ & -0.0 & 1831 & \\
\hline C & 588 & 624 & \\
\hline$P$ & 2.64 & 9.364 & 23.46 \\
\hline $\mathrm{C}$ & 2.87 & & 21.6 \\
\hline $\mathrm{C}$ & 3.001 & 10.4 & \\
\hline $\mathrm{C}$ & 3.23 & 10.3 & 19. \\
\hline C & 02 & & 18.8 \\
\hline C & 3.25 & & \\
\hline $\mathrm{C}$ & 3.00 & & \\
\hline C & 1.98 & & \\
\hline C & 2.70 & & 24. \\
\hline $\mathrm{C}$ & 1.92 & & \\
\hline C & 0.71 & & \\
\hline 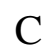 & 0.75 & & \\
\hline & 0.88 & & 25. \\
\hline C & -0.92 & 799 & 26.4 \\
\hline $\mathrm{C}$ & 0.025 & 05 & 27.3 \\
\hline 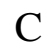 & 1.12 & & \\
\hline $\mathrm{C}$ & 0.8 & & \\
\hline C & -0.41 & & \\
\hline C & -0.0 & & 27. \\
\hline $\mathrm{C}$ & $-1.00 \mathrm{~s}$ & 4.49 & 4593 \\
\hline C & 259 & 092 & 8039 \\
\hline C & 61 & 99 & 4426 \\
\hline C & 0.658 & 998 & 29.39 \\
\hline$C$ & 0.74520072213190 & 4.95733841813360 & 28.90259259954535 \\
\hline $\mathrm{O}$ & -0.2 & 0927 & 32303 \\
\hline C & -1.22 & & 35844033 \\
\hline$H$ & -1.0 & & \\
\hline $\mathrm{H}$ & -1.045224437 & 1645990861 & 27.80423356656826 \\
\hline $\mathrm{H}$ & -2.26120642771942 & 0.92769539470525 & 29.01022055786197 \\
\hline & 1.30 & 3.32241928 & 30.2071 \\
\hline & רתר & 2.51046772061151 & \\
\hline
\end{tabular}




$\begin{array}{clcc}\text { H } & -1.65789250740089 & 4.79850421023949 & 26.50813365042147 \\ \text { H } & 1.46402698151665 & 5.64497403160685 & 29.34926448308688 \\ \text { H } & 1.50954273188332 & 9.74864611598175 & 26.74110694510800 \\ \text { H } & 2.03392813535632 & 7.47057284243229 & 28.08676591549163 \\ \text { H } & -0.88648980400859 & 9.48144103320878 & 25.51963093854632 \\ \text { H } & -1.86226340684118 & 7.02971261466745 & 26.12469838052139 \\ \text { H } & 3.65952471623374 & 7.03029888287748 & 25.21107449409342 \\ \text { H } & 2.16405946847713 & 4.78301877460114 & 25.54146891216695 \\ \text { H } & -0.09534294053218 & 5.06875341060810 & 24.08880448839513 \\ \text { H } & -0.00973003908577 & 7.49986890861748 & 22.87183162536741 \\ \text { H } & 2.90362531464845 & 11.39654115429796 & 21.39494652033844 \\ \text { H } & 2.90501810192503 & 7.08773841483493 & 21.54719720218483 \\ \text { H } & 3.31823727238052 & 11.31209866835323 & 18.95405696839100 \\ \text { H } & 3.35061147263682 & 7.00348643278185 & 19.10979574847490 \\ \text { H } & 3.54343346630288 & 9.11386440333279 & 17.79531097583826 \\ \text { H } & 2.20182737360854 & 11.52134972896883 & 25.19015578226465 \\ \text { H } & -1.88669176024634 & 11.37958049566411 & 22.38509220075722 \\ \text { H } & 0.35259712156694 & 13.13996097993297 & 25.61530611470569 \\ \text { H } & -1.70111637353036 & 13.04731005071527 & 24.19188305834513 \\ \text { H } & -2.03691153784934 & 9.39097212901786 & 21.31004785770484 \\ \text { H } & -0.82944733459672 & 8.81212032879335 & 20.11290634691237 \\ \text { H } & -1.08134976715039 & 10.57341931097650 & 20.34648312438932\end{array}$

$3 b$

$E=-4320.72931518 \mathrm{Eh}$

P 8.34481203110057

O 8.40758495855281

2.67222214283284

5.19175897008106

Ru 8.89838499312372

O 5.35408549367668

$-4.14501865050630$

$-0.98112573992510$

3.99164021462675

7.02332322539166

3.22036693023916

5.30861225603736

Fe 10.61515108663343

0.26667970984727

3.99556440465275

Cl 10.32014796446371

5.11278163985957

5.44375090698990

Cl 10.64374703614256

2.40143507181657

7.47010223355298

C 8.96403711661567

5.72928342975187

8.43835515302906

H 9.79726236771260

6.42507026476470

8.53173935833296

C 7.95784465175768

3.52849152618222

8.98591291480198

C 9.61849605257231

2.04350947080722

4.06901173677674

C 7.39079202524061

1.15200365872032

5.56225710316445

C 11.80194207453382

$-1.39498874998220$

3.98872520392039

H 12.63282111327692

$-1.54472119171060$

3.30513213100900

C 6.94963547462021

4.99430889594246

7.24948210830366 


\begin{tabular}{|c|c|c|c|}
\hline & & & \\
\hline & & & \\
\hline & & & \\
\hline & & 284 & \\
\hline & & 9533 & 554 \\
\hline & 7943451 & 852715 & 68659 \\
\hline & 085 & 3164 & 7329 \\
\hline & & & \\
\hline & & & \\
\hline C & & -2. & 039 \\
\hline & 2138939583 & 4573836788 & 13482739 \\
\hline & 903 & 37393 & 2802 \\
\hline & & 3.6 & 4.0 \\
\hline & & & \\
\hline $\mathrm{C}$ & & & \\
\hline & & & 999 \\
\hline & & & \\
\hline $\mathrm{H}$ & 06 & & 077 \\
\hline & & & 739 \\
\hline & & & \\
\hline ᄃ & 51 & 249 & 1198 \\
\hline $\mathrm{H}$ & & 714 & 5756 \\
\hline C & 44 & 0.5 & 36282 \\
\hline & 72 & & 0087 \\
\hline C & & 76 & 11 \\
\hline $\mathrm{H}$ & & -1 & 23 \\
\hline C & & & 2.2 \\
\hline & & 5.8 & 77422 \\
\hline c & 8 & 321 & 2289 \\
\hline $\mathrm{H}$ & & 686 & 5.00 \\
\hline $\mathrm{C}$ & & -3. & \\
\hline C & 10.19 & 24887 & 7546475 \\
\hline $\mathrm{H}$ & 1504 & 86007 & 1793 \\
\hline 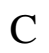 & 117 & & 5819 \\
\hline $\mathrm{H}$ & 10.6 & 55958 & 1.20790043785779 \\
\hline C & 71986 & 301928208 & 1.605621380073 \\
\hline $\mathrm{H}$ & 03604053 & -3.11667034662760 & 581064909 \\
\hline $\mathrm{C}$ & 5.85453238561039 & -0.61654235751114 & 4.93555212474689 \\
\hline $\mathrm{H}$ & & $-1 .($ & 391341 \\
\hline & & & \\
\hline & & & 6.95129681060724 \\
\hline
\end{tabular}




$\begin{array}{llll}\text { H } & 7.99405943206590 & 8.48077258128871 & 8.44220183054561 \\ \text { H } & 6.39582490720735 & 8.23787651629302 & 7.70079233824415 \\ \text { C } & 7.31464681998113 & 7.15752032483068 & 5.27105544096940 \\ \text { H } & 7.48134917103822 & 8.08350226786869 & 4.70342965942611 \\ \text { H } & 6.22649887350273 & 7.03386197637609 & 5.37959851319052 \\ \text { H } & 7.70934188638350 & 6.32265308999691 & 4.67956417849071 \\ \text { C } & 10.55818794298909 & -0.69186857722988 & 5.80917643397736 \\ \text { H } & 10.28672663667792 & -0.19515055029092 & 6.73478630559738 \\ \text { C } & 8.00035814437284 & 2.26486087655551 & 9.79006845214153 \\ \text { H } & 7.23209852080771 & 1.55668844090437 & 9.45769876798961 \\ \text { H } & 7.82818615908419 & 2.48386566554041 & 10.85523064030820 \\ \text { H } & 8.98878488529310 & 1.79003212962449 & 9.69446403072608 \\ \text { C } & 11.87678700990332 & -0.74316705344573 & 5.26155628992171 \\ \text { H } & 12.76701801627439 & -0.31404869999513 & 5.71168594070803 \\ \text { C } & 6.50741106277922 & 0.57959424830395 & 4.63640015325171 \\ \text { H } & 6.31917690166189 & 1.07735961006167 & 3.68431977898056 \\ \text { C } & 7.01447508735546 & -4.10369897143072 & -1.27397886435547 \\ \text { H } & 6.65480896173429 & -3.06613831540321 & -1.37846491451722 \\ \text { H } & 6.42346183204617 & -4.61517225410223 & -0.49560463917515 \\ \text { H } & 6.89206573105720 & -4.62897687185937 & -2.22760603422105 \\ \text { C } & 5.18663971387428 & 4.62481569912063 & 3.32614545771906 \\ \text { H } & 4.11812100841771 & 4.77578505483798 & 3.46875340667930 \\ \text { C } & 6.95380332218340 & -0.68198955126978 & 7.08828906610542 \\ \text { H } & 7.14343016850693 & -1.17833002771161 & 8.04112463408374 \\ \text { C } & 6.07352922368798 & -1.24991990830423 & 6.16387683527036 \\ \text { H } & 5.56435941513576 & -2.18656555629193 & 6.39442982783623 \\ \text { C } & 11.58641832487595 & 1.70185449669559 & 2.89279062583681 \\ \text { H } & 12.64110544959934 & 1.67791742299934 & 2.63307525767363 \\ \text { C } & 3.94037821184898 & 3.29272610811076 & 5.47123783274110 \\ \text { H } & 3.60553549984013 & 4.32919092957358 & 5.64318931081586 \\ \text { H } & 3.71221648142288 & 2.68325148289458 & 6.35215096046926 \\ \text { H } & 3.41641966825070 & 2.87998750875566 & 4.59463957072035\end{array}$

$4 \mathrm{~b}$

$\mathrm{E}=-6846.77822738 \mathrm{Eh}$

$\begin{array}{lll}\text { Ru } 8.71293888281913 & 2.39364700820905 & 2.11351330090849\end{array}$

C $7.92512952436997 \quad-7.77858401686881 \quad-2.99571437806632$

H $9.01538741668450 \quad-7.64286243300148 \quad-3.09322356692973$

H $7.64712981915803 \quad-8.77085573427532 \quad-3.36768475226919$

H $7.64303865945725 \quad-7.69332341668194 \quad-1.93279585339428$ 

O 7.21023939372898
$-6.84571879443637$
$-3.80072472908355$
Fe 6.84423882543181
$-0.29787706829778$
$-1.32892980307448$
Cl 7.86183070354839
3.42677537128044
0.13126859009559
P 8.10032366542747
0.31670122415550
1.82326294198220
C 7.39139092972379
$-5.51442821583854$
$-3.53017725209446$
O 10.40816887645778
1.67095183497625
0.70210951376693
Fe 7.75457559620212
3.36687526162585
6.65065851620531
Cl 10.24216335636654
1.75994693058901
3.83824529825563
P 7.02800874606306
3.14042581157957
3.28672475411132
C 6.79671117478464
$-3.24916459088379$
$-4.14094544986616$
H 6.24511313885090
$-2.56920705560057$
O 9.16192898005371
0.40631383314143
$-4.79128578345395$
C 6.67800008366671
$-4.61887694143568$
12.84761618151835
H 6.03951903334814
$-5.02101385593829$
$-4.34093514344388$
O 9.47260989492997
4.53363646481440
$-5.12812224871091$
C 8.22540919641319
$-5.01298445810777$
2.57858463749616
H 8.79014837053827
$-5.68458259397914$
$-2.52027091507848$
C 8.33043959741476
$-3.63457136416890$
$-1.87560922576746$
H 8.96836956873456
$-3.26025079160955$
$-2.33062736402863$
C 7.62149884723170
$-2.72298529939636$
$-1.52875305525602$
C 8.71710655866676
$-0.61957330440098$
$-3.12840064451734$
H 9.53688393533175
$-1.10745691203438$
$-2.08634368424091$
C 7.72729091394306
$-1.27908484236989$
$-1.56981548057411$
C 7.25925096562638
1.00638555673974
$-2.89997039319198$
H 6.76355651988884
1.96333918995782
$-2.85339932777908$
C 8.42624693172088
0.78008892600321
$-2.98578457720790$
H 8.92638125922704
1.54402727723470
$-2.05975858435012$
C 6.82501558565908
$-0.25595091901410$
$-1.47372411180046$
H 5.93568746429313
$-0.42133205964724$
$-3.37069401129666$
C 4.90773690734526
$-0.10051137970478$
$-3.97206155773427$
0.27587414086524
$-0.67486986730783$
H 4.08217713882231
C 5.33333266415660
$-1.46717239398554$
$-1.27205456655256$
H 4.89623579781977
$-2.31148883846198$
$-0.58936735872827$
C 6.48698695725343
$-1.51866135673504$
$-1.11572162457901$
H 7.05216668099782
$-2.41124105996534$
0.25208945060553
C 5.79304602167702
0.69815195594583
0.49895957500578
H 5.78746058359530
1.77661700114208
0.11024085231260
C 9.60198923480226
$-0.50560694793589$
0.20858481077178
C 6.77855086236070
$-0.17264085242310$
1.15839295401470
C 9.77383740537474
$-1.89237090207104$
0.69470074032777
H 9.01155691691562
$-2.52944971270204$
1.12558009697842
1.57423680575456 


\begin{tabular}{|c|c|c|c|}
\hline & 692 & -2.46488298011490 & \\
\hline & 1 & 174647202 & \\
\hline & & & \\
\hline H & 5677 & 801 & 5 \\
\hline & .88406029453325 & 639215 & 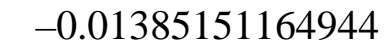 \\
\hline & 5262035 & -2.0 & 973 \\
\hline & 460 & 20 & \\
\hline & 843 & & \\
\hline & 28 & & \\
\hline & 0581716 & 34 & -0 \\
\hline & 6673 & 08 & \\
\hline & & & \\
\hline $\mathrm{H}$ & & & 221 \\
\hline C & & & \\
\hline & 13 & -1.8 & \\
\hline$H$ & 00 & -2.2 & \\
\hline & 88 & 76 & \\
\hline $\mathrm{H}$ & 90 & - & \\
\hline $\mathrm{C}$ & & & \\
\hline $\mathrm{H}$ & 75 & -2.5 & \\
\hline & & & \\
\hline $\mathrm{C}$ & 94 & -0.9 & 3.6 \\
\hline $\mathrm{H}$ & & -0. & 691 \\
\hline 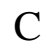 & & & 77 \\
\hline $\mathrm{H}$ & & & \\
\hline & & & \\
\hline $\mathrm{H}$ & 024 & & 1.12 \\
\hline( & & & 4924 \\
\hline $\mathrm{H}$ & & & 76 \\
\hline C & & & 7653 \\
\hline $\mathrm{H}$ & 69653 & & 0.99 \\
\hline & 7.26 & & 3245 \\
\hline & & & 3634 \\
\hline 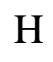 & & & 7552 \\
\hline $\mathrm{C}$ & & & \\
\hline - & 161250 & 154 & 1622324454 \\
\hline $\mathrm{C}$ & 6.5 & 15 & 277884 \\
\hline $\mathrm{H}$ & & & \\
\hline & & & 3.661317 \\
\hline & & & 3.89 \\
\hline & S & 2.69897195800991 & 5.01646455 \\
\hline
\end{tabular}




\begin{tabular}{|c|c|c|c|}
\hline & & & \\
\hline & 11.41498935776836 & 3.99078607451569 & 1557 \\
\hline & .12379120039044 & & 4836 \\
\hline & & & \\
\hline & 17196 & 151 & 136 \\
\hline & 5883 & 17 & \\
\hline & 29703 & $21 \varepsilon$ & 923 \\
\hline & 30 & 50 & \\
\hline & & 18 & \\
\hline & & & 528 \\
\hline & 7525 & 2.54 & 58557 \\
\hline & 4211 & 1.600 & 5.7209 \\
\hline 11 & 00 & 0.96188 & 297 \\
\hline & & 39 & \\
\hline & & 36 & 25 \\
\hline & & 78 & \\
\hline & & & \\
\hline & 33 & 3808 & 73 \\
\hline & 28 & 36 & \\
\hline & & & \\
\hline & 7.5 & 9 & 76 \\
\hline & & & \\
\hline & 43 & 23 & 127 \\
\hline & 287 & 74 & 15 \\
\hline & 19 & 6 & 27 \\
\hline C & 9.1 & & 59 \\
\hline & 9.62 & 80 & \\
\hline & 404 & 2447 & 0.5 \\
\hline & 75327 & 53297 & 9.42 \\
\hline $\mathrm{H}_{2}$ & 9.95 & 34 & 991 \\
\hline $\mathrm{C}$ & 8.58 & 25 & 01308 \\
\hline & 8.19 & $2.9757 \mathrm{c}$ & 12.7315 \\
\hline 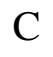 & 28543 & 33033 & 10.645607 \\
\hline & & & 1667 \\
\hline & .6 & & \\
\hline & & & \\
\hline H & 9.203 & 1.77586 & 14.43385717784508 \\
\hline & 8.77989832560751 & 0.07194298358967 & 14.80395450892092 \\
\hline
\end{tabular}




\section{HPLC Chromatograms}

\section{1-((SP)-(2-Biphenylyl)(phenyl)phosphine P-borane)-1'-(4-methoxyphenyl)ferrocene (1a)}

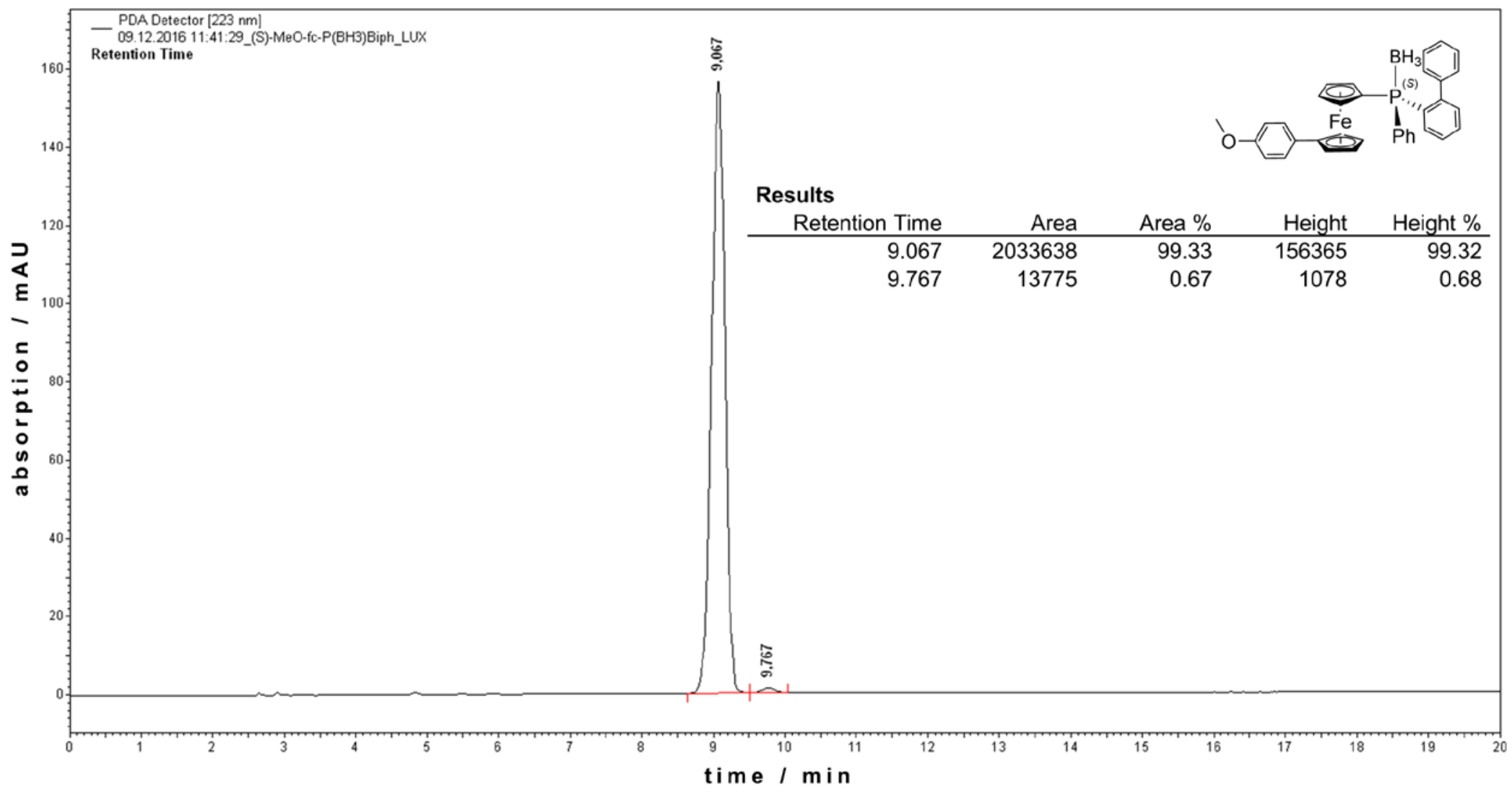

Figure S5. HPLC Chromatogram of 1 a. 
1-((SP)-(2-Methoxyphenyl)(phenyl)phosphine P-borane)-1'-(4-methoxyphenyl)ferrocene (1b)

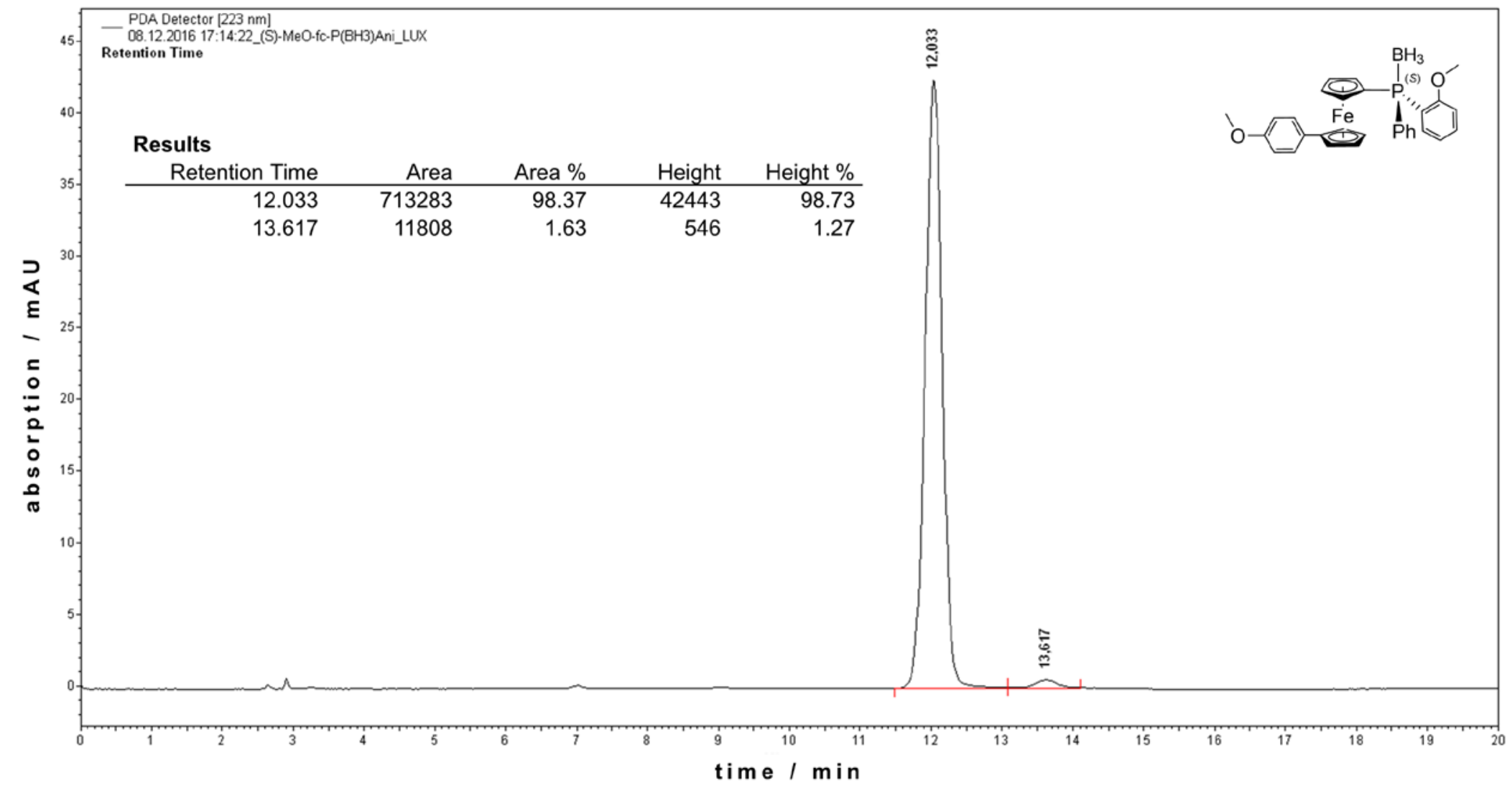

Figure S6. HPLC Chromatogram of $\mathbf{1 b}$. 


\section{1-((SP)-(2-Biphenylyl)(phenyl)phosphine)-1'-(4-methoxyphenyl)ferrocene (2a)*}

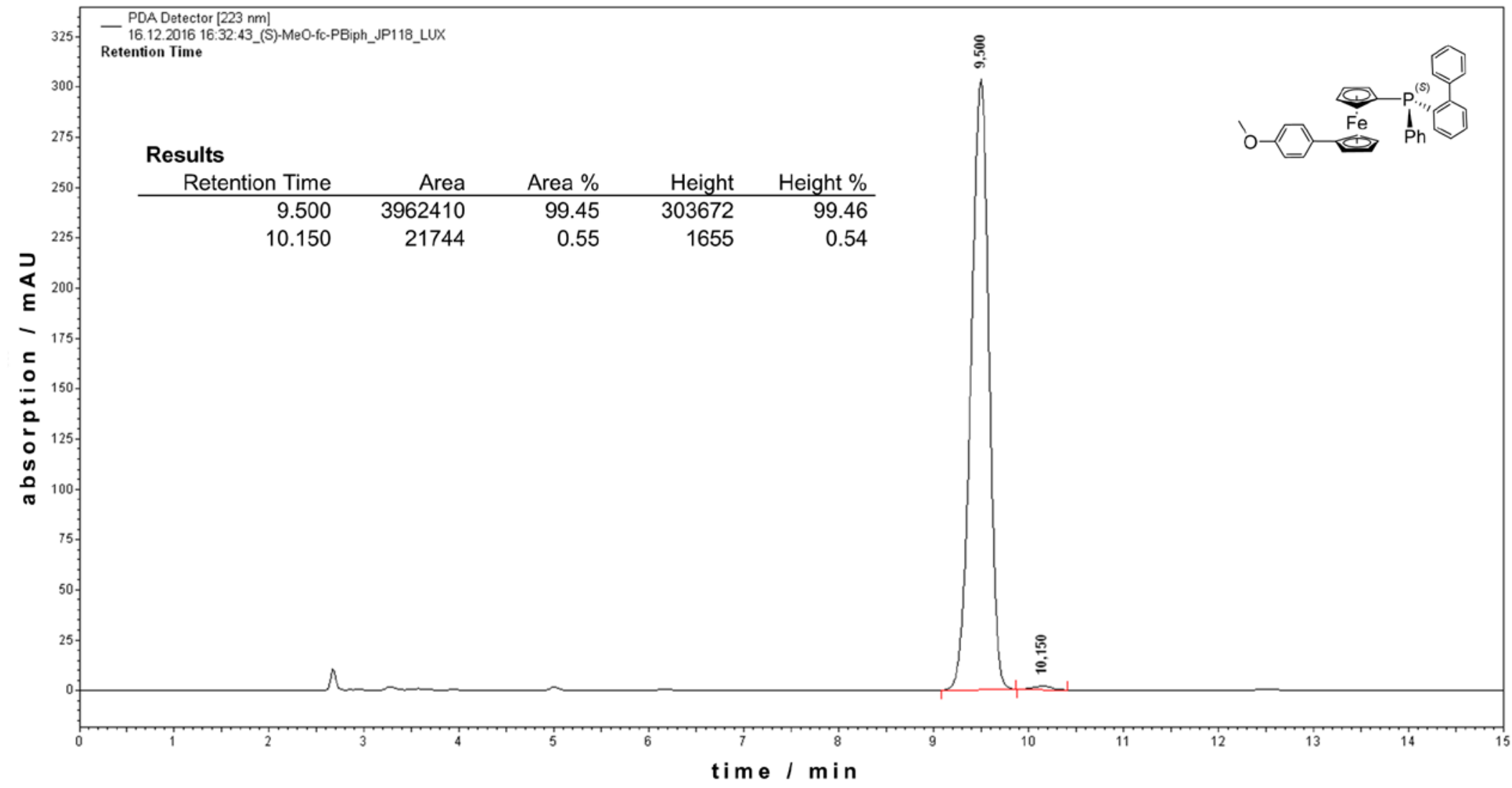

Figure S7. HPLC Chromatogram of $\mathbf{2 a .}$

${ }^{*}$ Determined after reprotection by reaction with $\mathrm{BH}_{3} \cdot \mathrm{SMe}_{2}$ 
1-((SP)-(2-Methoxyphenyl)(phenyl)phosphine)-1'-(4-methoxyphenyl)ferrocene (2b)*

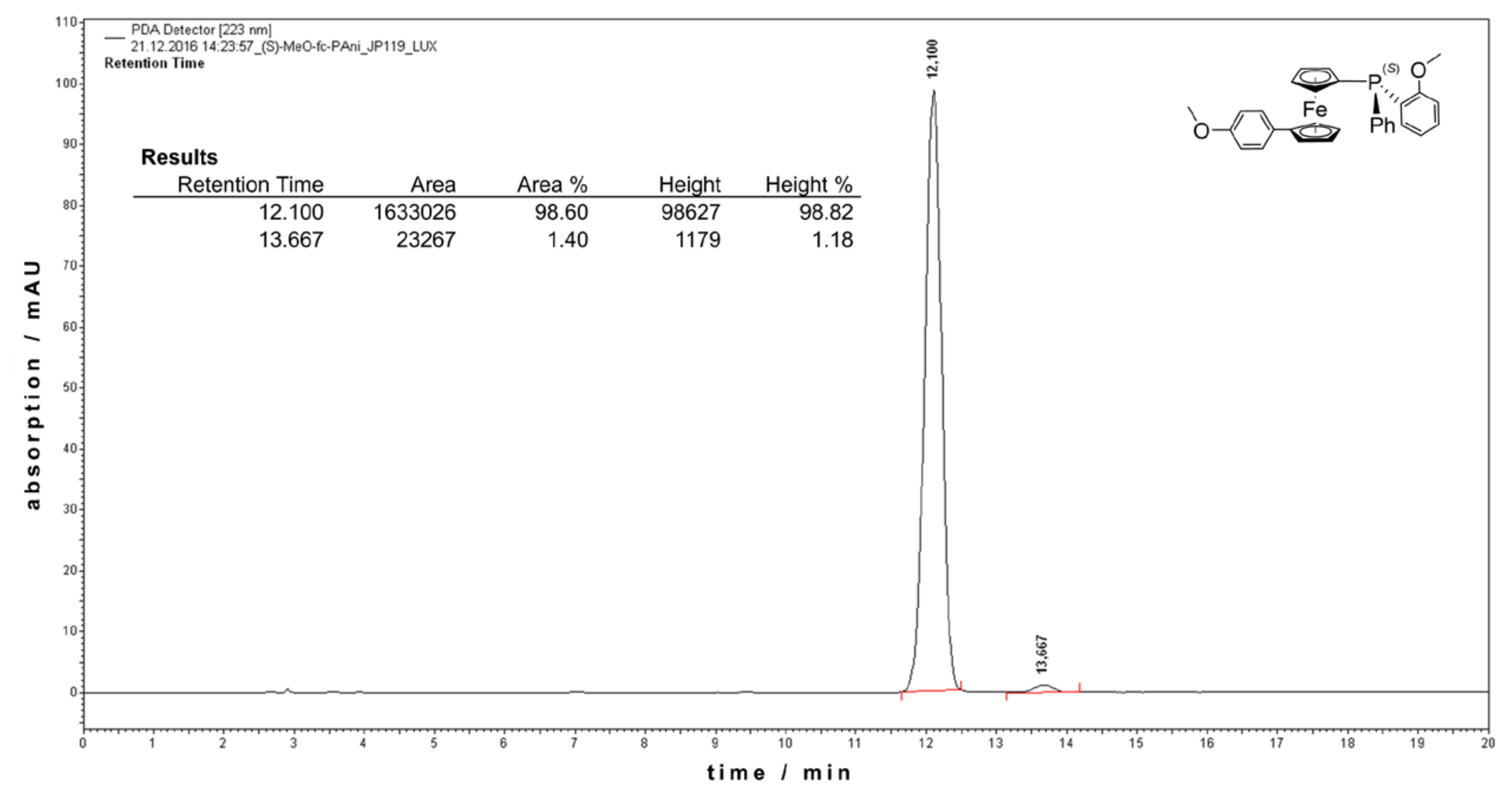

Figure S8. HPLC Chromatogram of $\mathbf{2 b .}$

${ }^{*}$ Determined after reprotection by reaction with $\mathrm{BH}_{3} \cdot \mathrm{SMe}_{2}$ 


\section{NMR Spectra}

1-((SP)-(2-Biphenylyl)(phenyl)phosphine P-borane)-1'-(4-methoxyphenyl)ferrocene (1a)

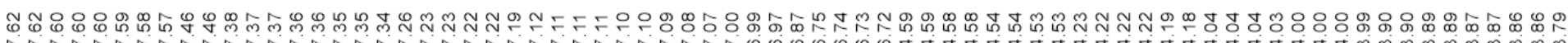

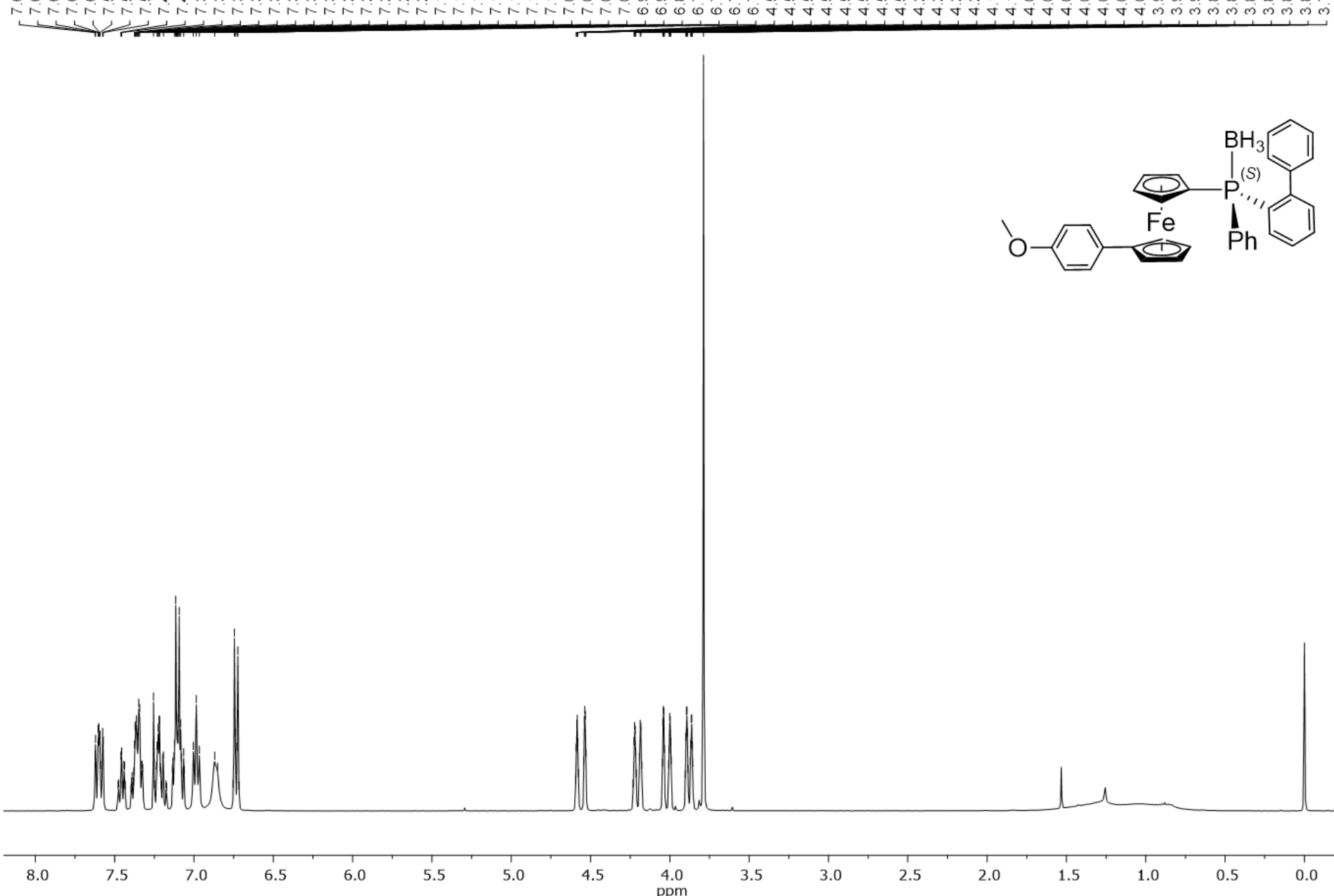

Figure S9. ${ }^{1} \mathrm{H}-\mathrm{NMR}$ spectrum of $\mathbf{1 a}$. 


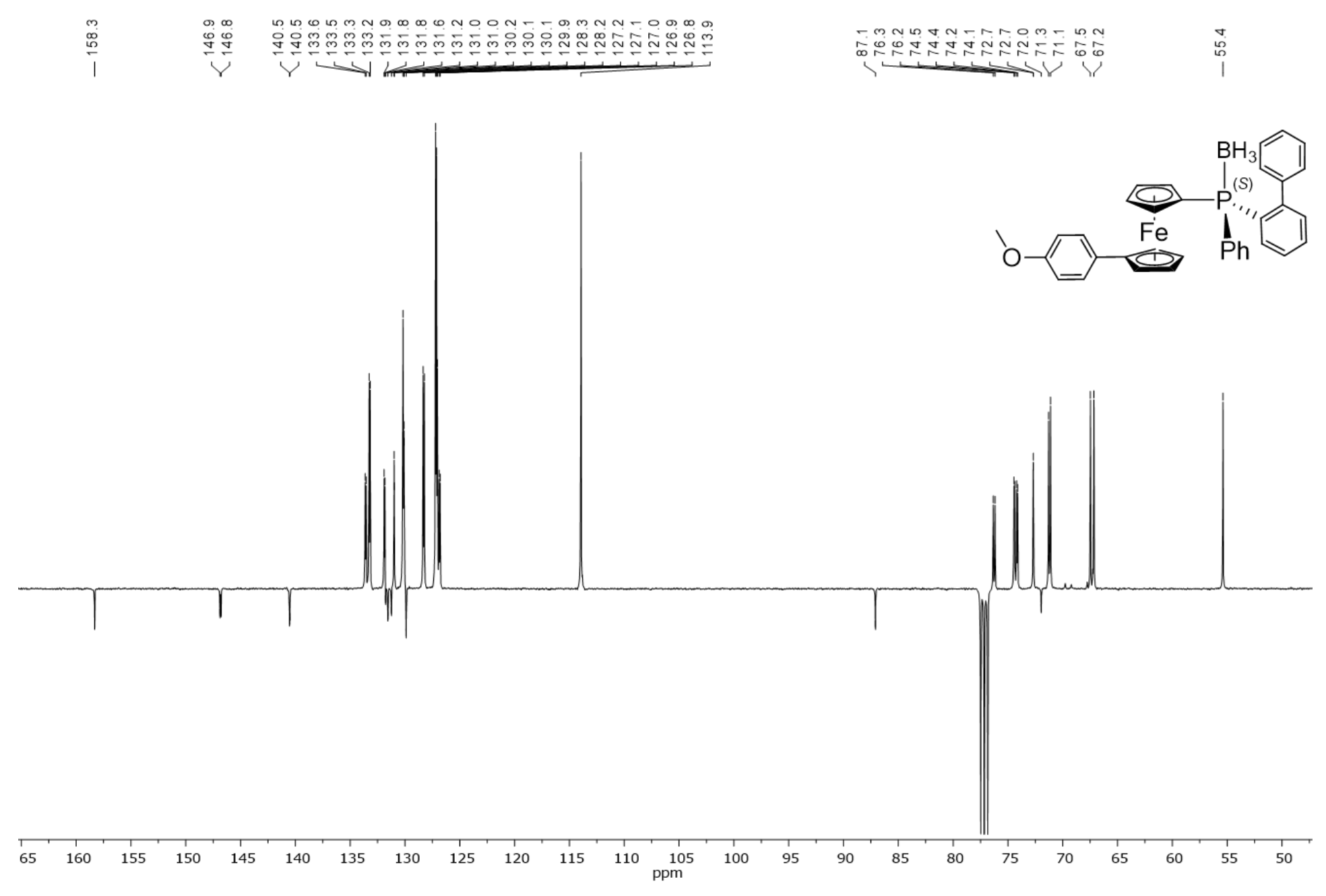

Figure S10. ${ }^{13} \mathrm{C}\left\{{ }^{1} \mathrm{H}\right\}$-NMR spectrum of 1a. 


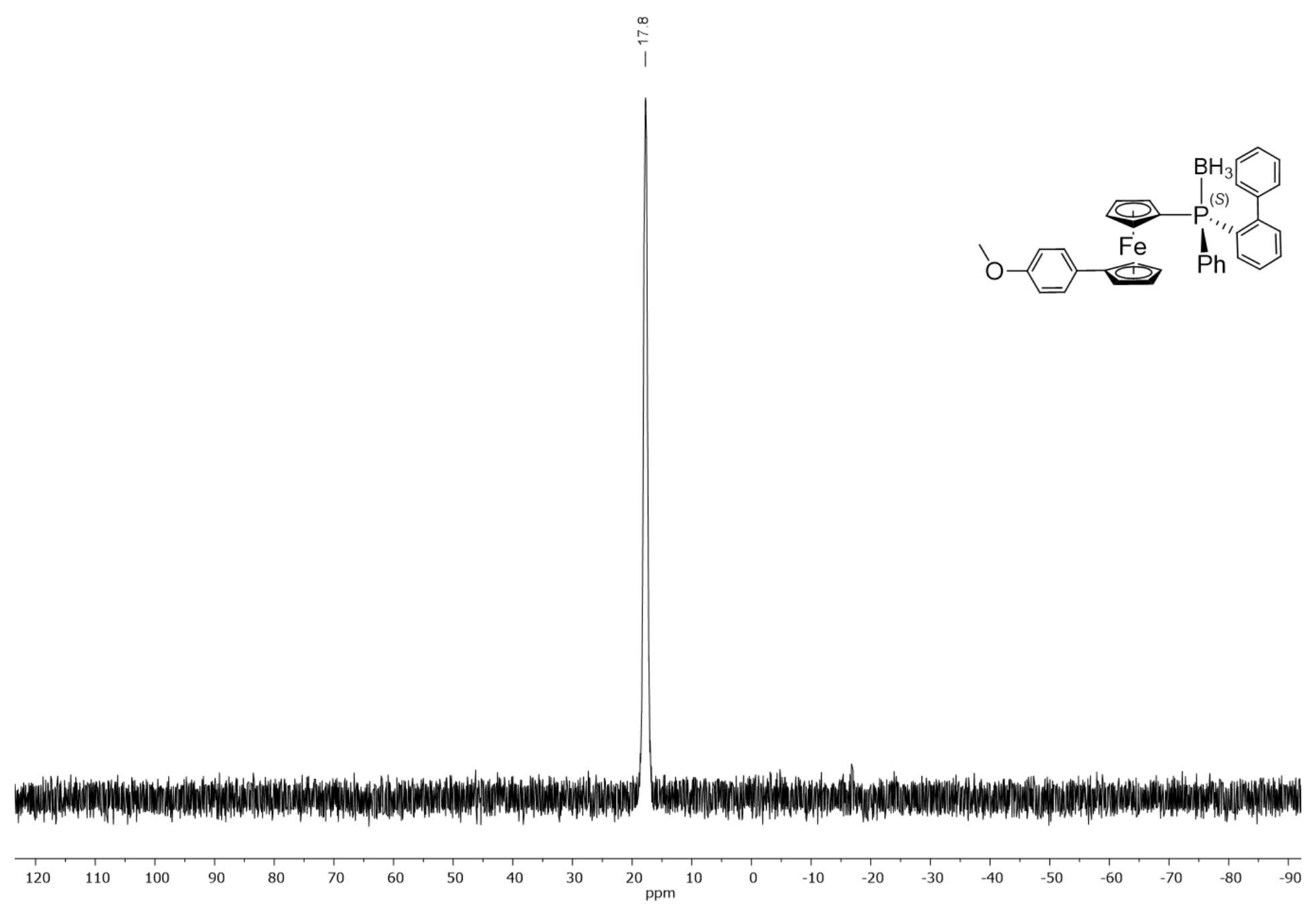

Figure S11. ${ }^{31} \mathrm{P}\left\{{ }^{1} \mathrm{H}\right\}$-NMR spectrum of $\mathbf{1 a}$. 
1-((SP)-(2-Methoxyphenyl)(phenyl)phosphine P-borane)-1'-(4-methoxyphenyl)ferrocene (1b)

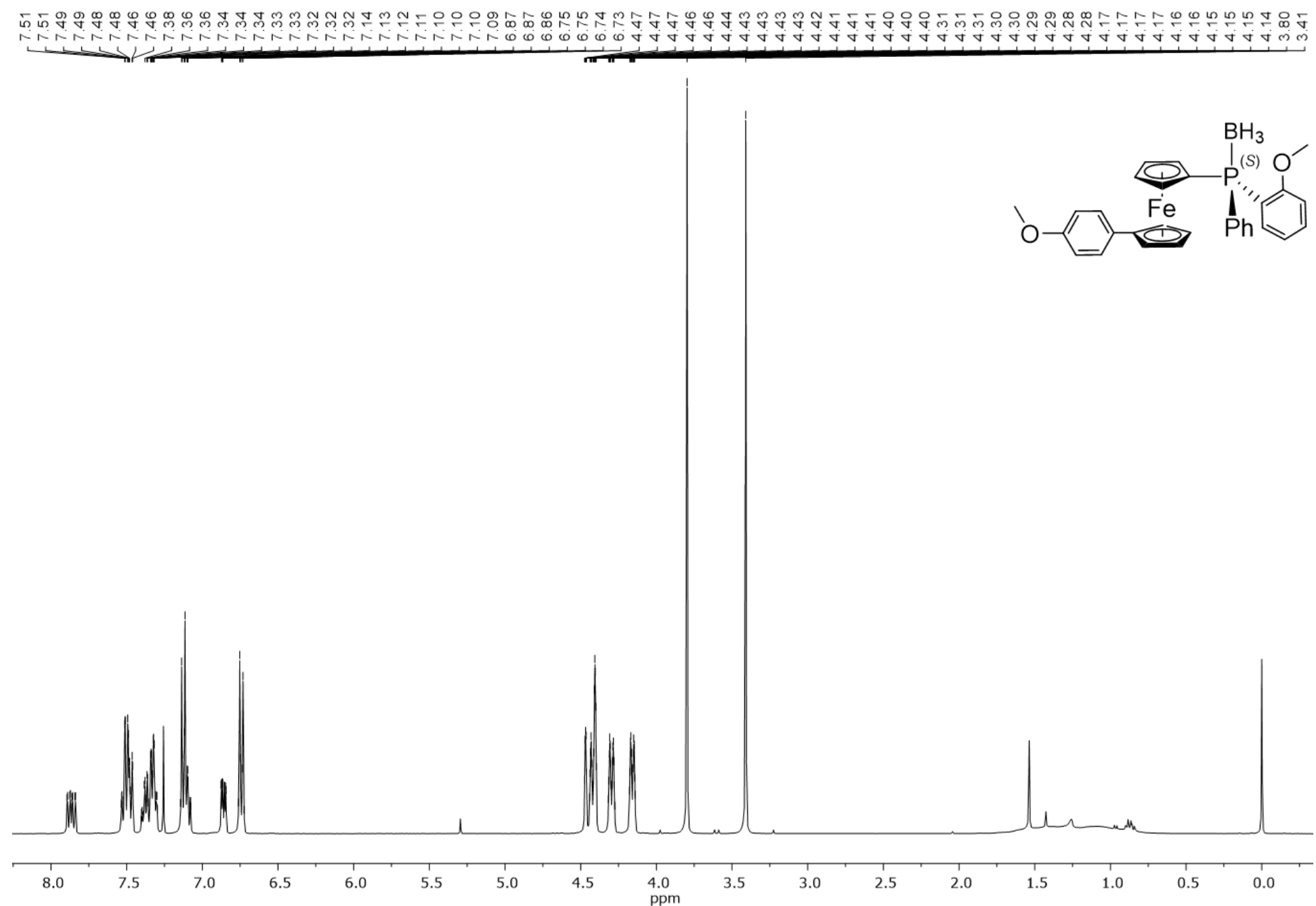

Figure S12. ${ }^{1} \mathrm{H}-\mathrm{NMR}$ spectrum of $\mathbf{1 b}$. 


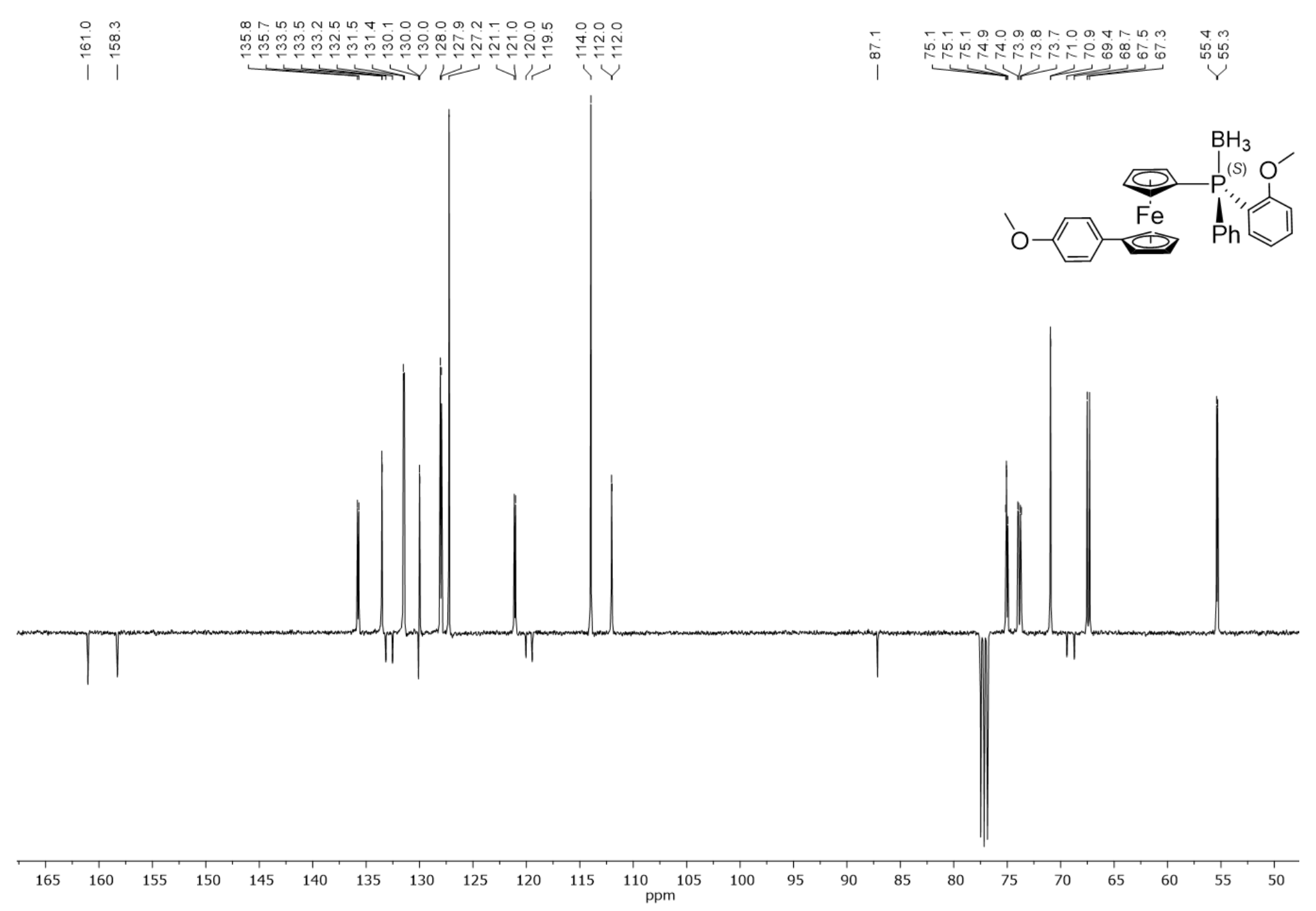

Figure S13. ${ }^{13} \mathrm{C}\left\{{ }^{1} \mathrm{H}\right\}$-NMR spectrum of $\mathbf{1 b}$. 


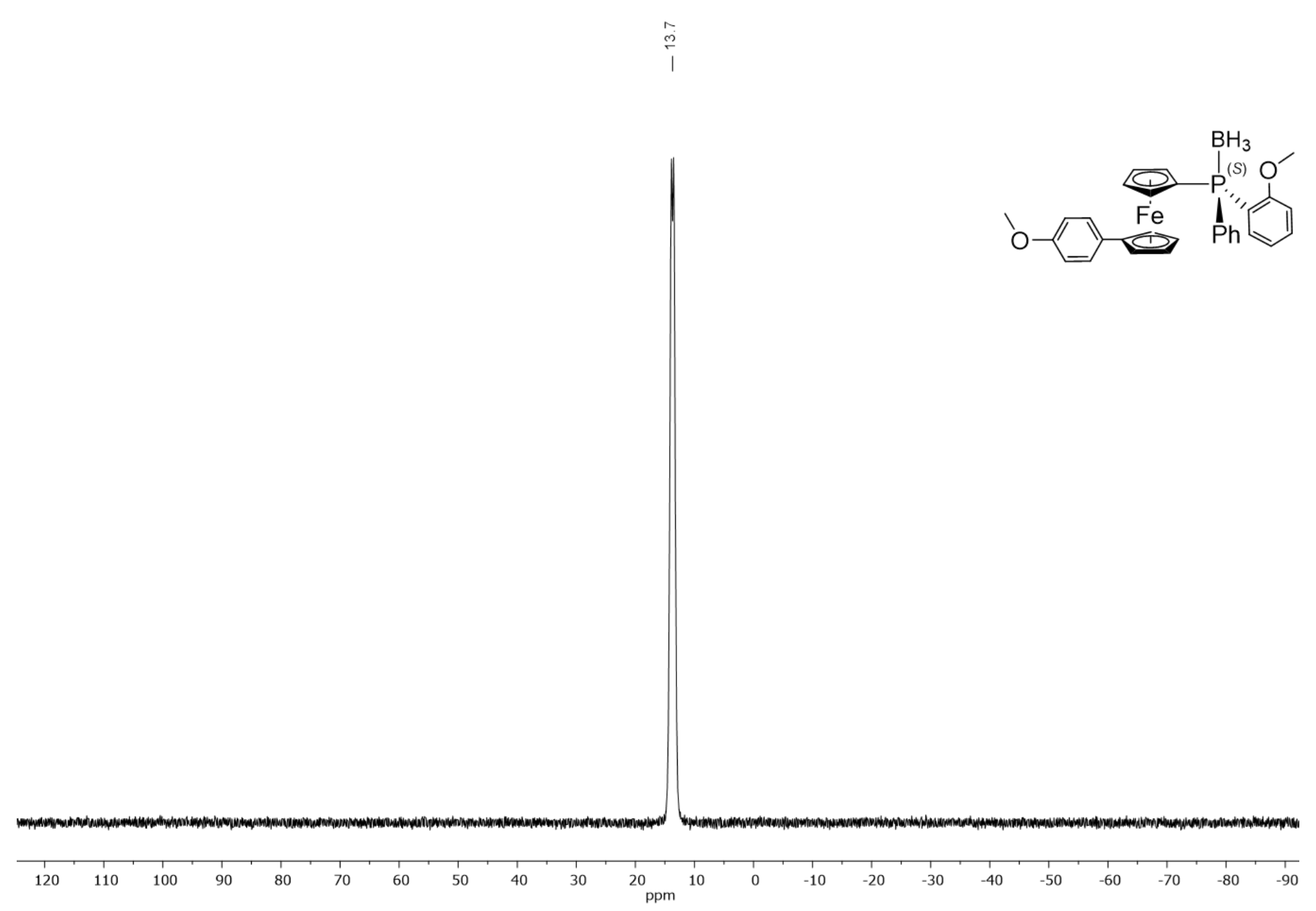

Figure S14. ${ }^{31} \mathrm{P}\left\{{ }^{1} \mathrm{H}\right\}$-NMR spectrum of $\mathbf{1 b}$. 
1-((SP)-(2-Biphenylyl)(phenyl)phosphine)-1'-(4-methoxyphenyl)ferrocene (2a)

-

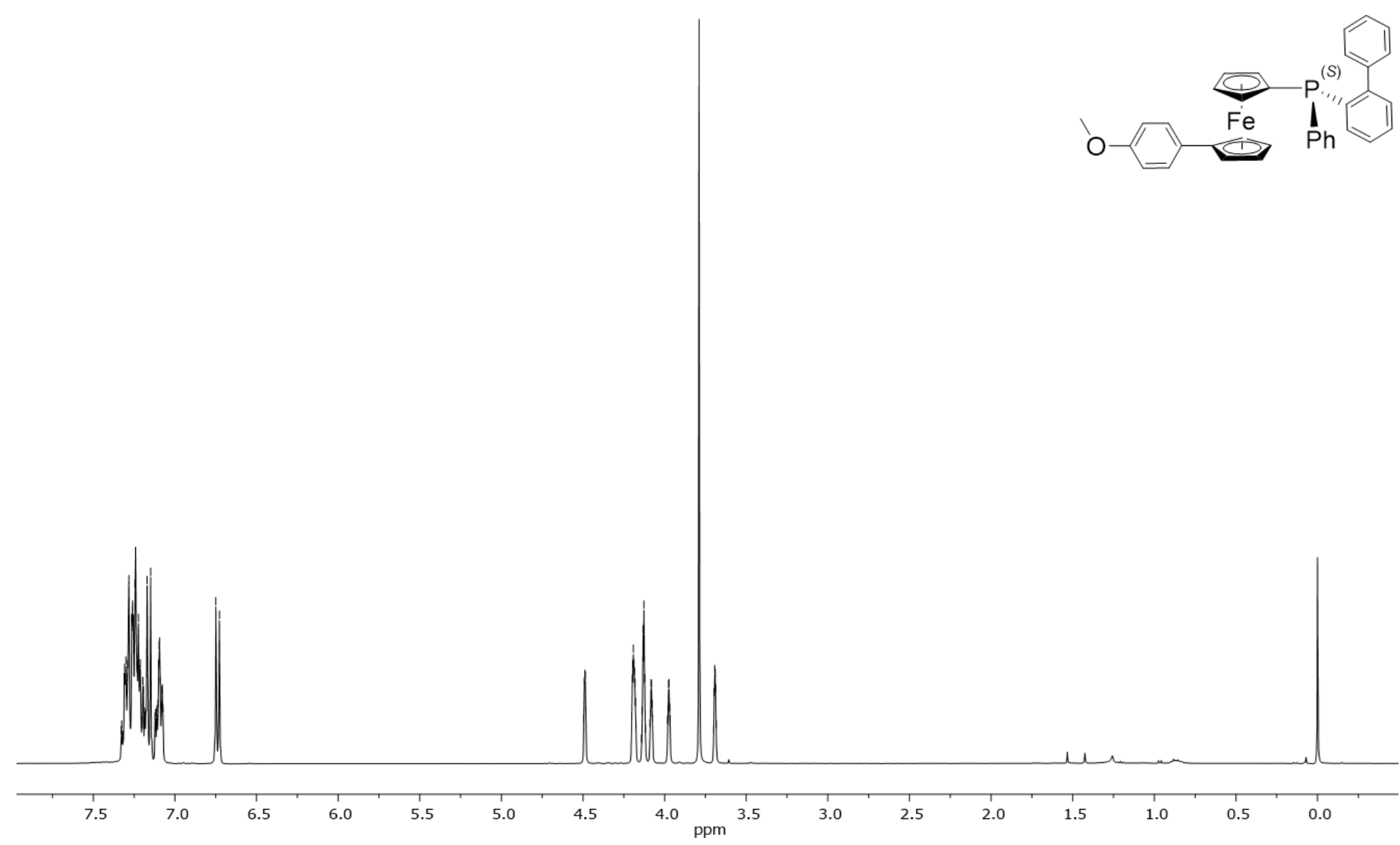

Figure S15. ${ }^{1} \mathrm{H}-\mathrm{NMR}$ spectrum of $2 \mathrm{a}$. 


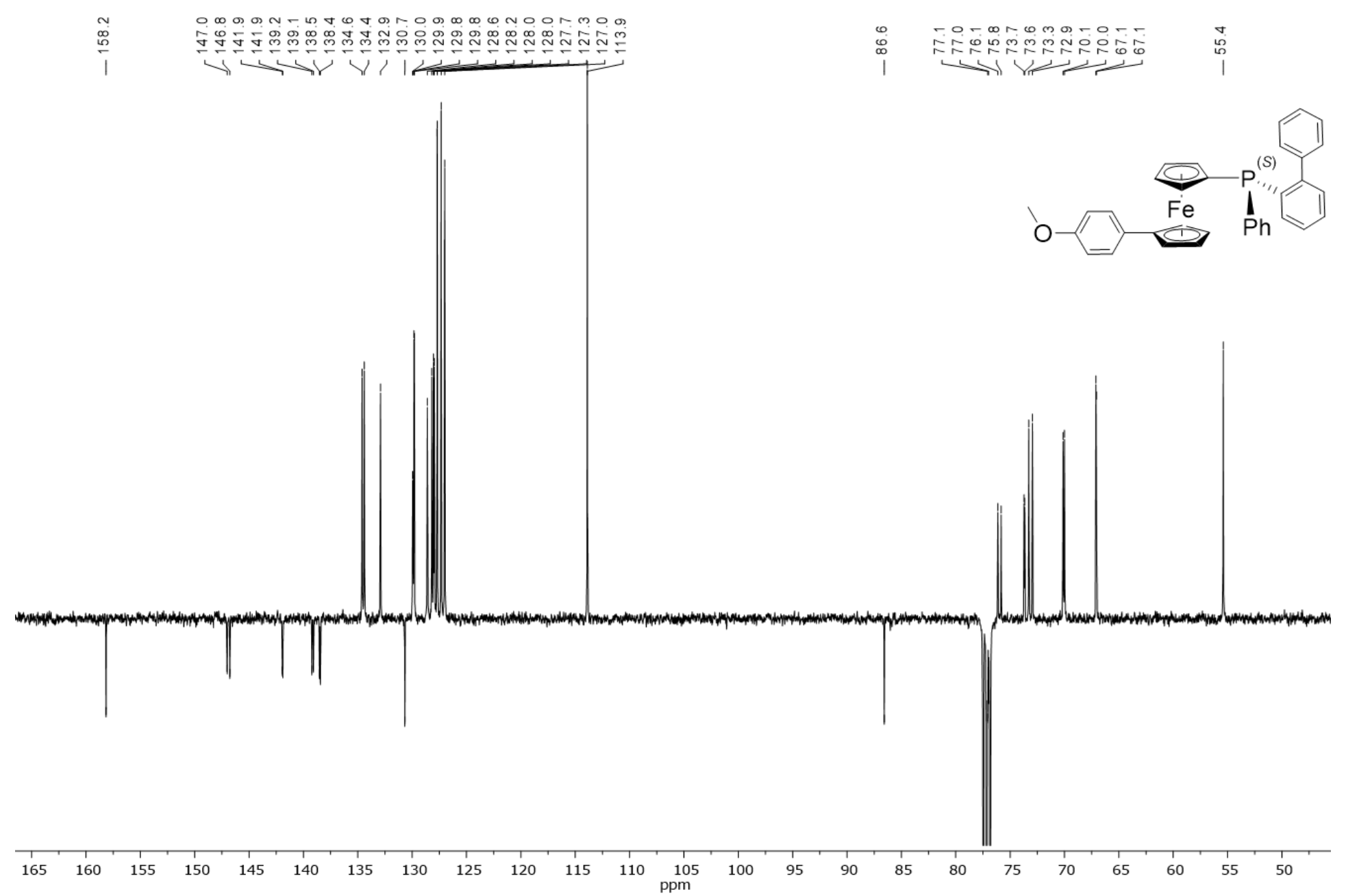

Figure S16. ${ }^{13} \mathrm{C}\left\{{ }^{1} \mathrm{H}\right\}$-NMR spectrum of 2a. 


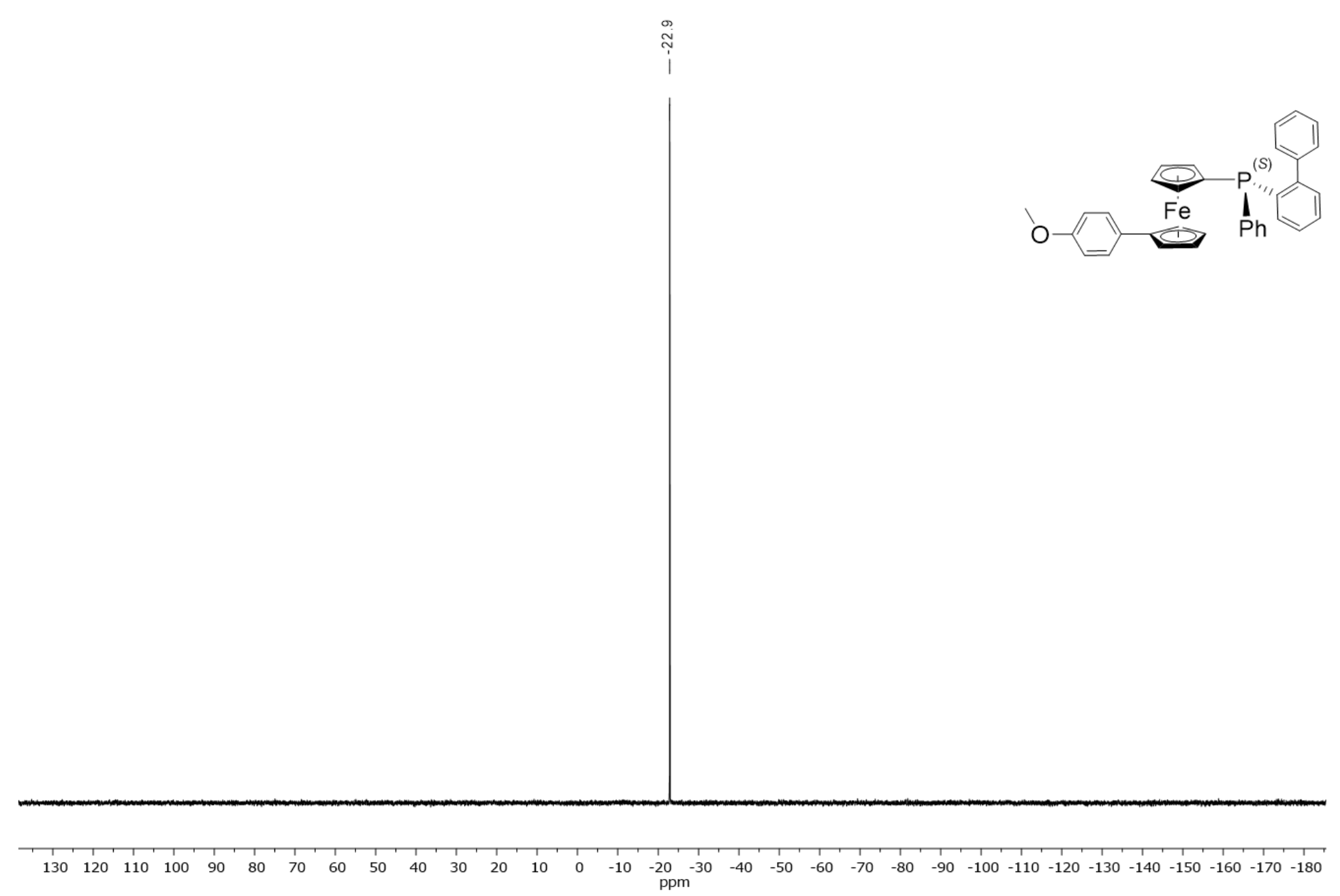

Figure S17. ${ }^{31} \mathrm{P}\left\{{ }^{1} \mathrm{H}\right\}$-NMR spectrum of $\mathbf{2 a}$. 
1-((SP)-(2-Methoxyphenyl)(phenyl)phosphine)-1'-(4-methoxyphenyl)ferrocene (2b)

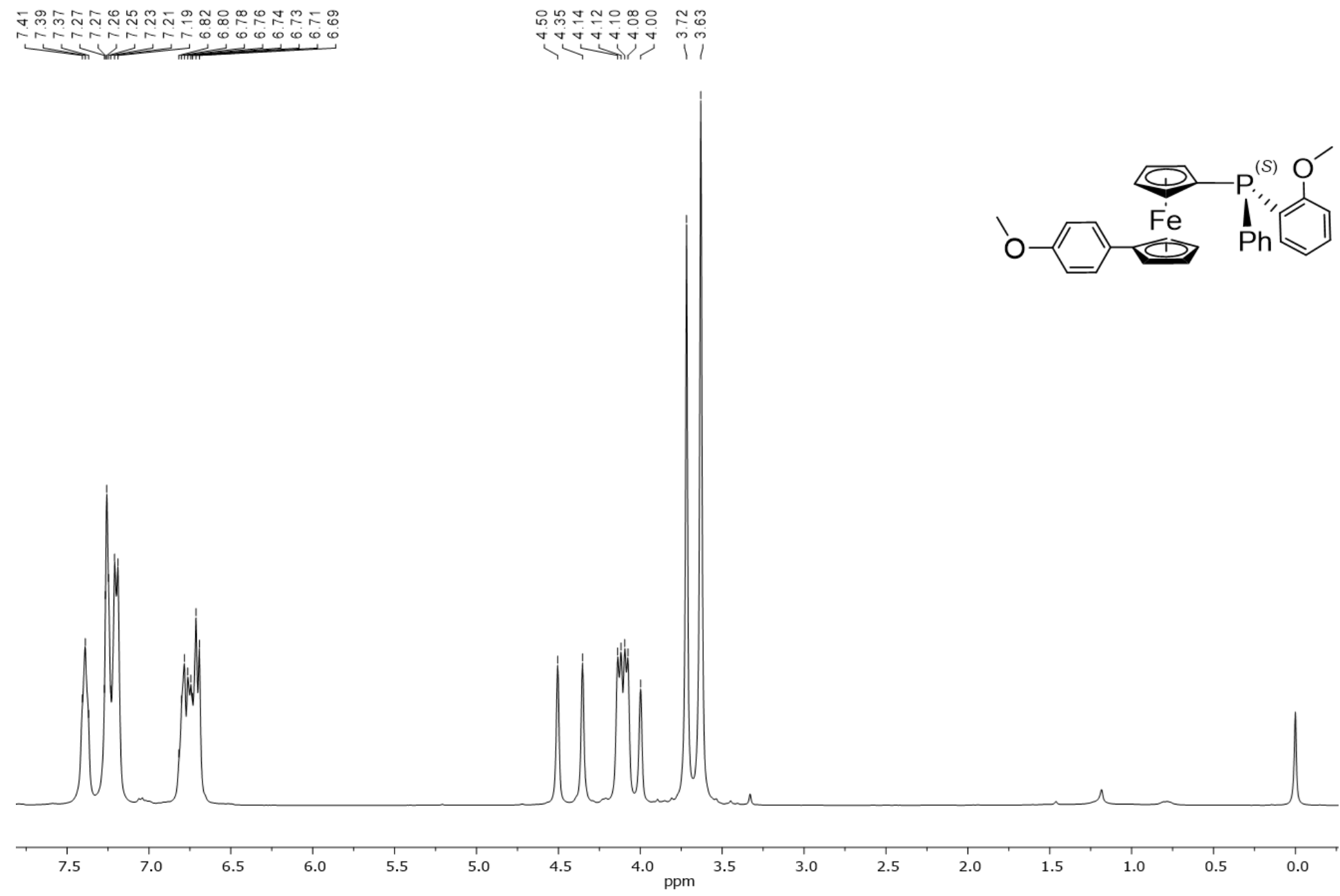

Figure S18. ${ }^{1} \mathrm{H}-\mathrm{NMR}$ spectrum of $\mathbf{2 b}$. 


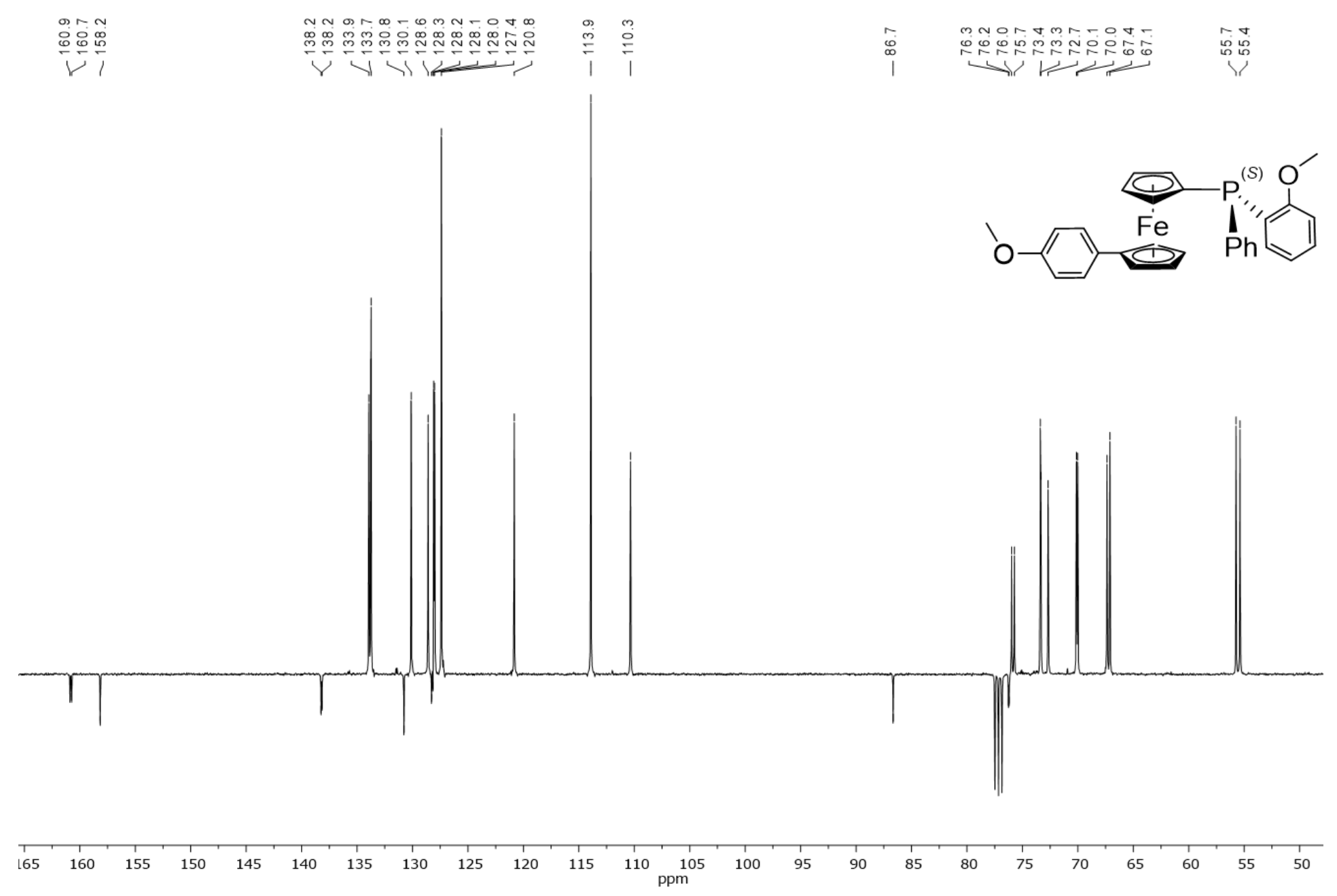

Figure S19. ${ }^{13} \mathrm{C}\left\{{ }^{1} \mathrm{H}\right\}$-NMR spectrum of $\mathbf{2 b}$. 


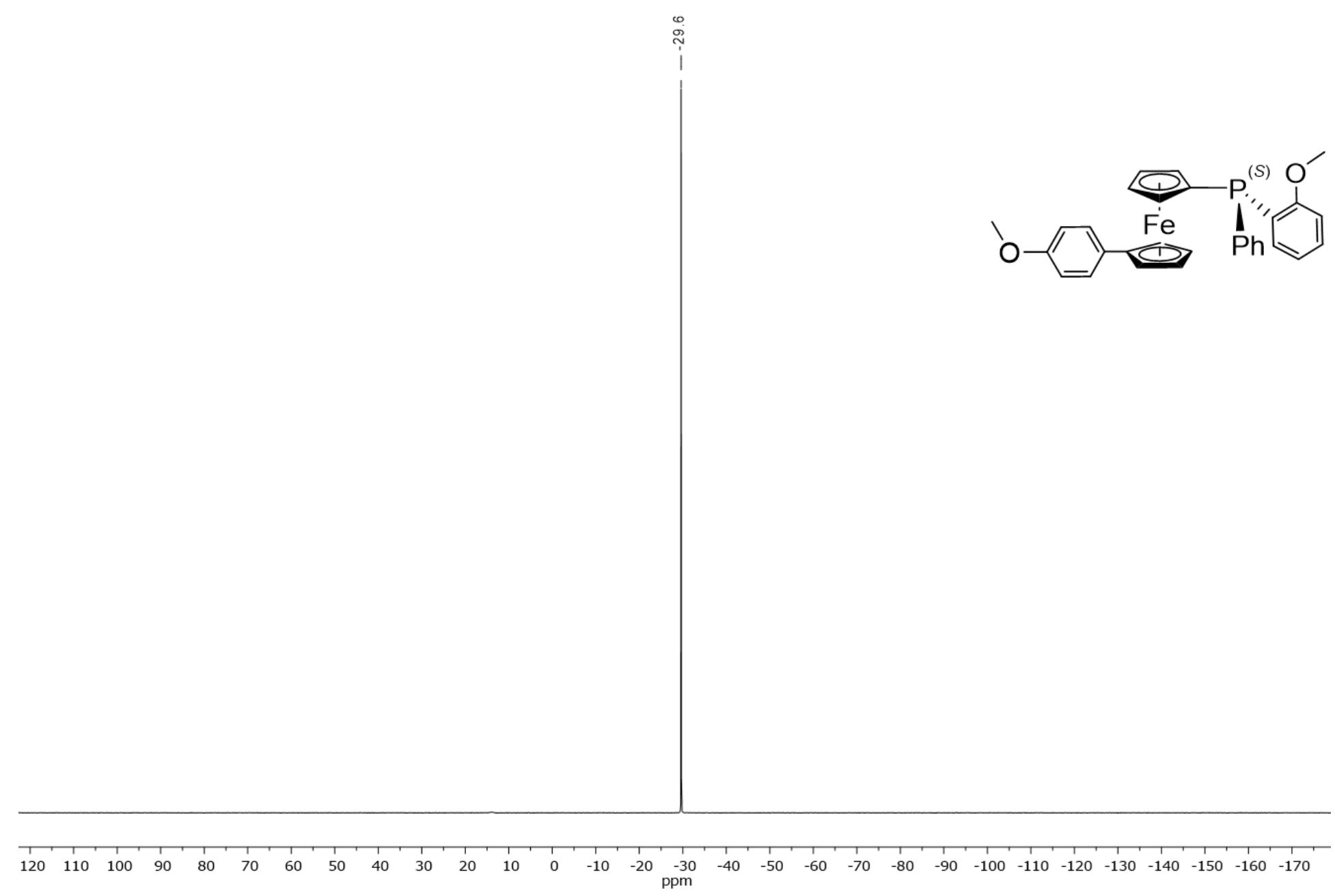

Figure S20. ${ }^{31} \mathrm{P}\left\{{ }^{1} \mathrm{H}\right\}$-NMR spectrum of $\mathbf{2 b}$. 
1-((SP)-(2-Methoxyphenyl)(phenyl)phosphino)[( $\eta^{6}-p$-cymene)dichlororuthenium(II)]-1'-(4-methoxyphenyl)ferrocene (3b)

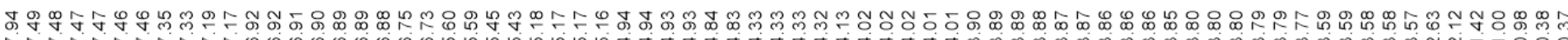
$\longrightarrow$ 1

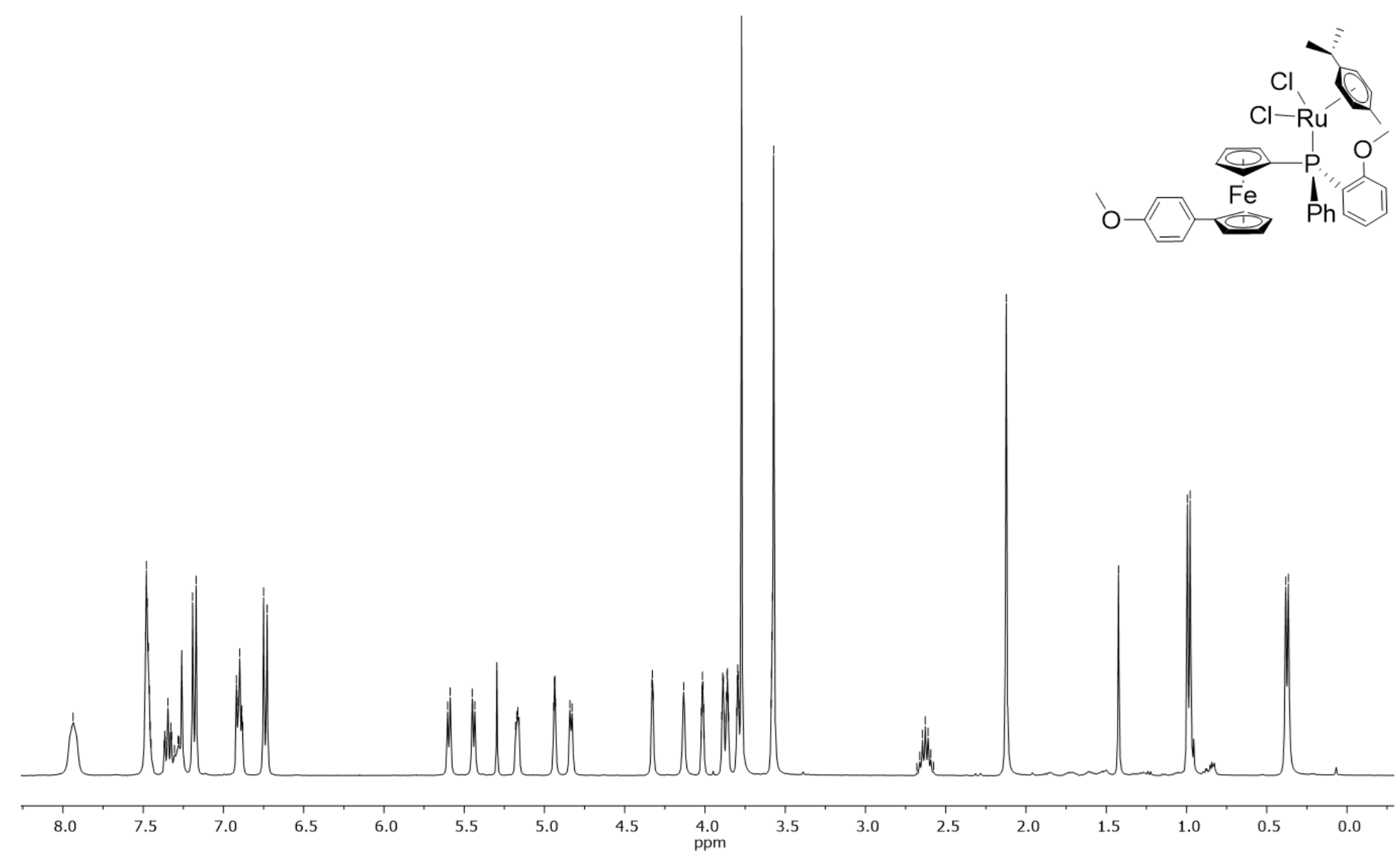

Figure S21. ${ }^{1} \mathrm{H}-\mathrm{NMR}$ spectrum of $\mathbf{3 b}$. 


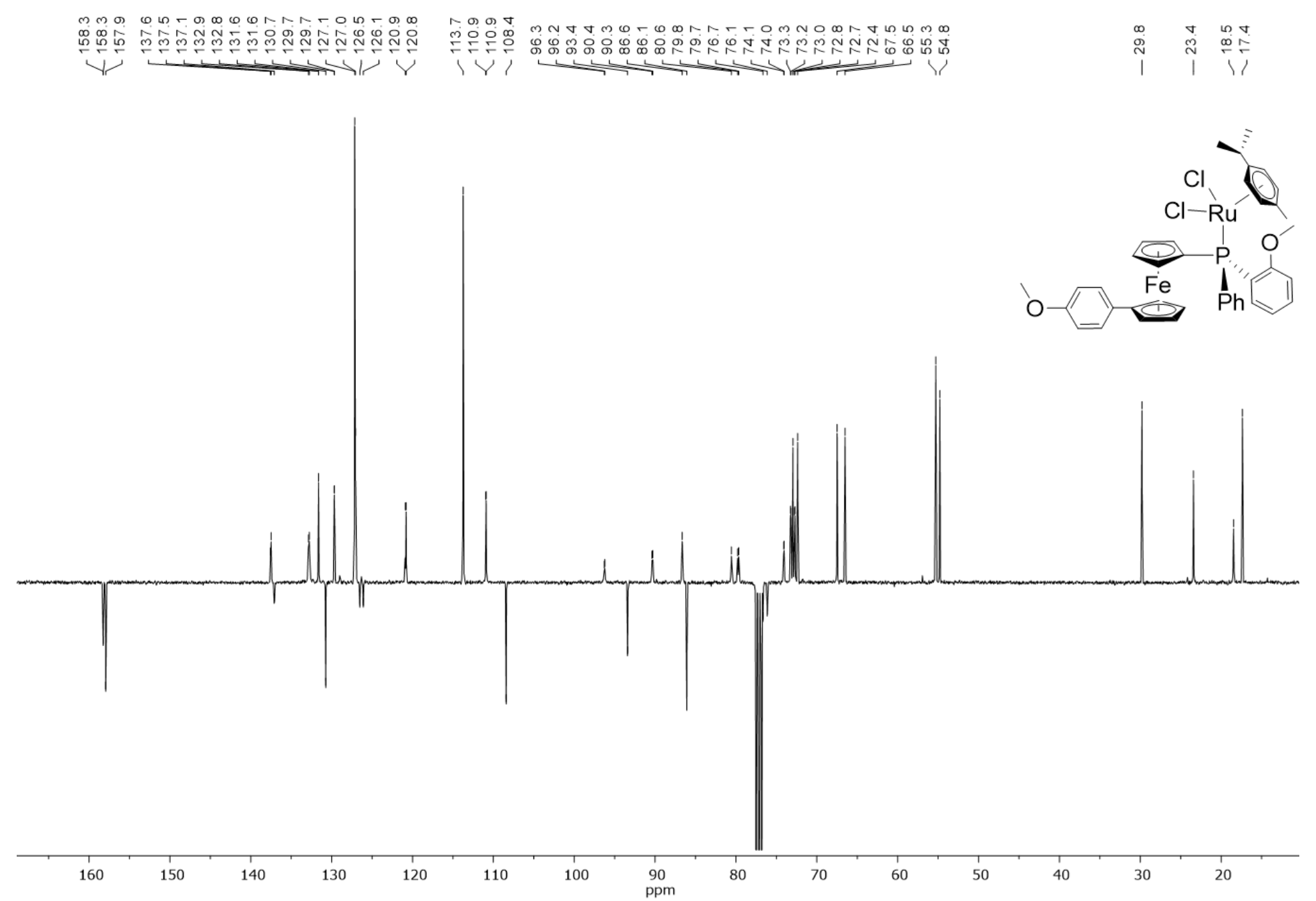

Figure S22. ${ }^{13} \mathrm{C}\left\{{ }^{1} \mathrm{H}\right\}$-NMR spectrum of $\mathbf{3 b}$. 


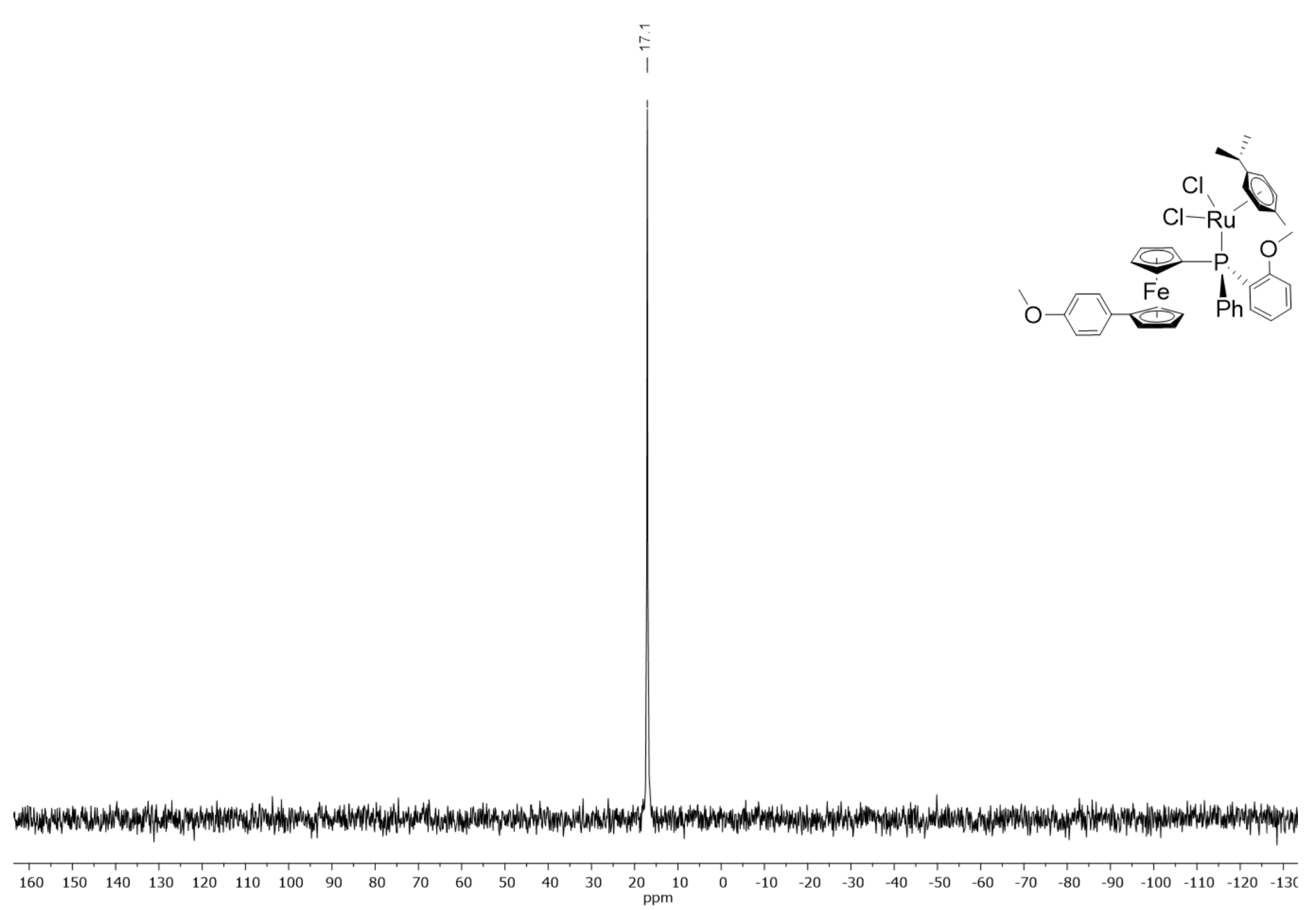

Figure S23. ${ }^{31} \mathrm{P}\left\{{ }^{1} \mathrm{H}\right\}$-NMR spectrum of $\mathbf{3 b}$. 


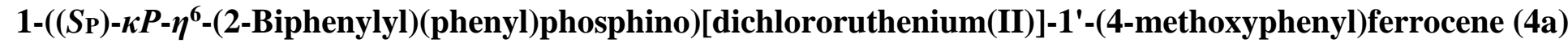

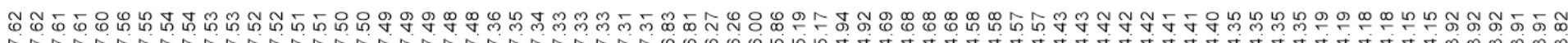

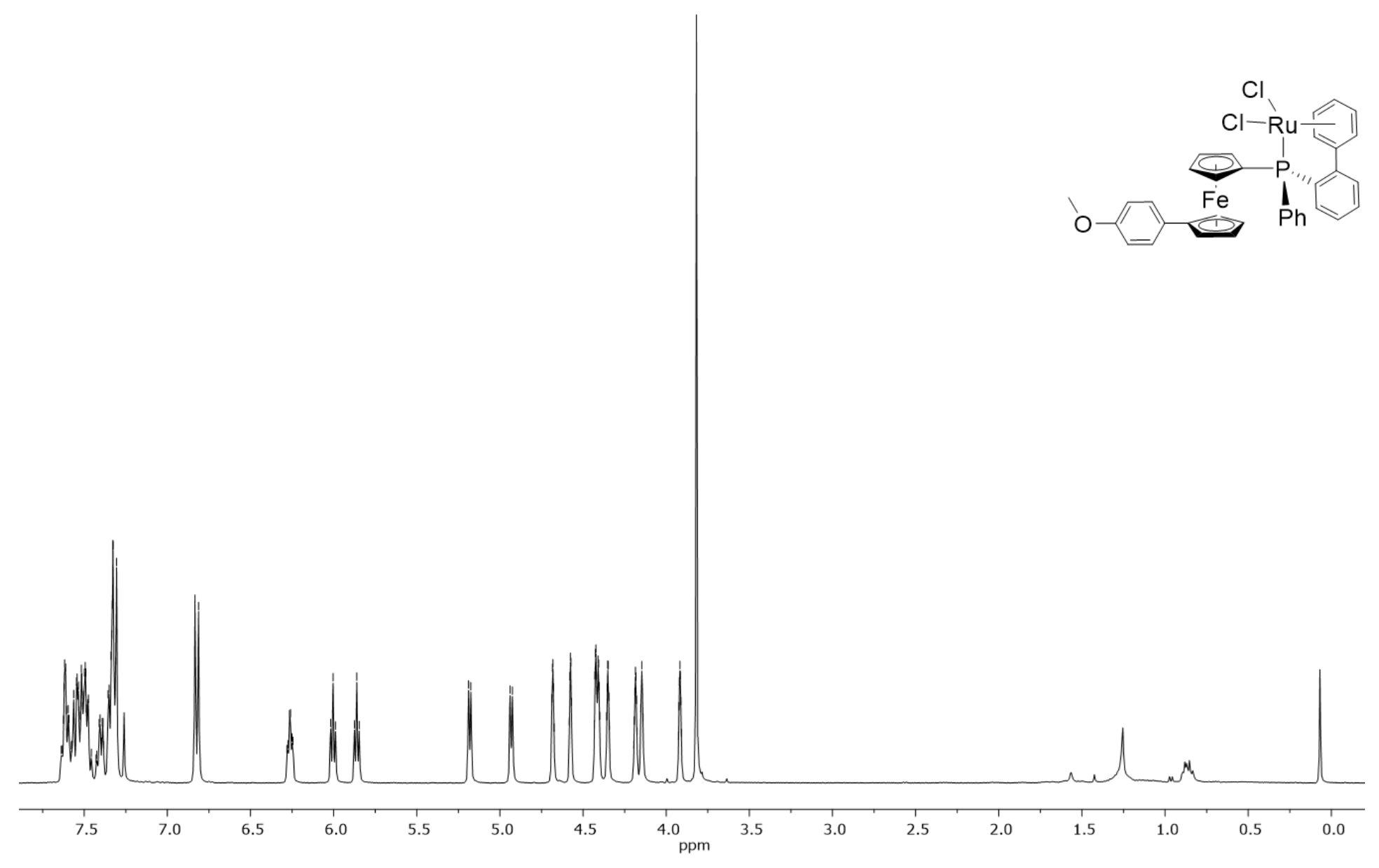

Figure S24. ${ }^{1} \mathrm{H}-\mathrm{NMR}$ spectrum of $\mathbf{4 a}$. 


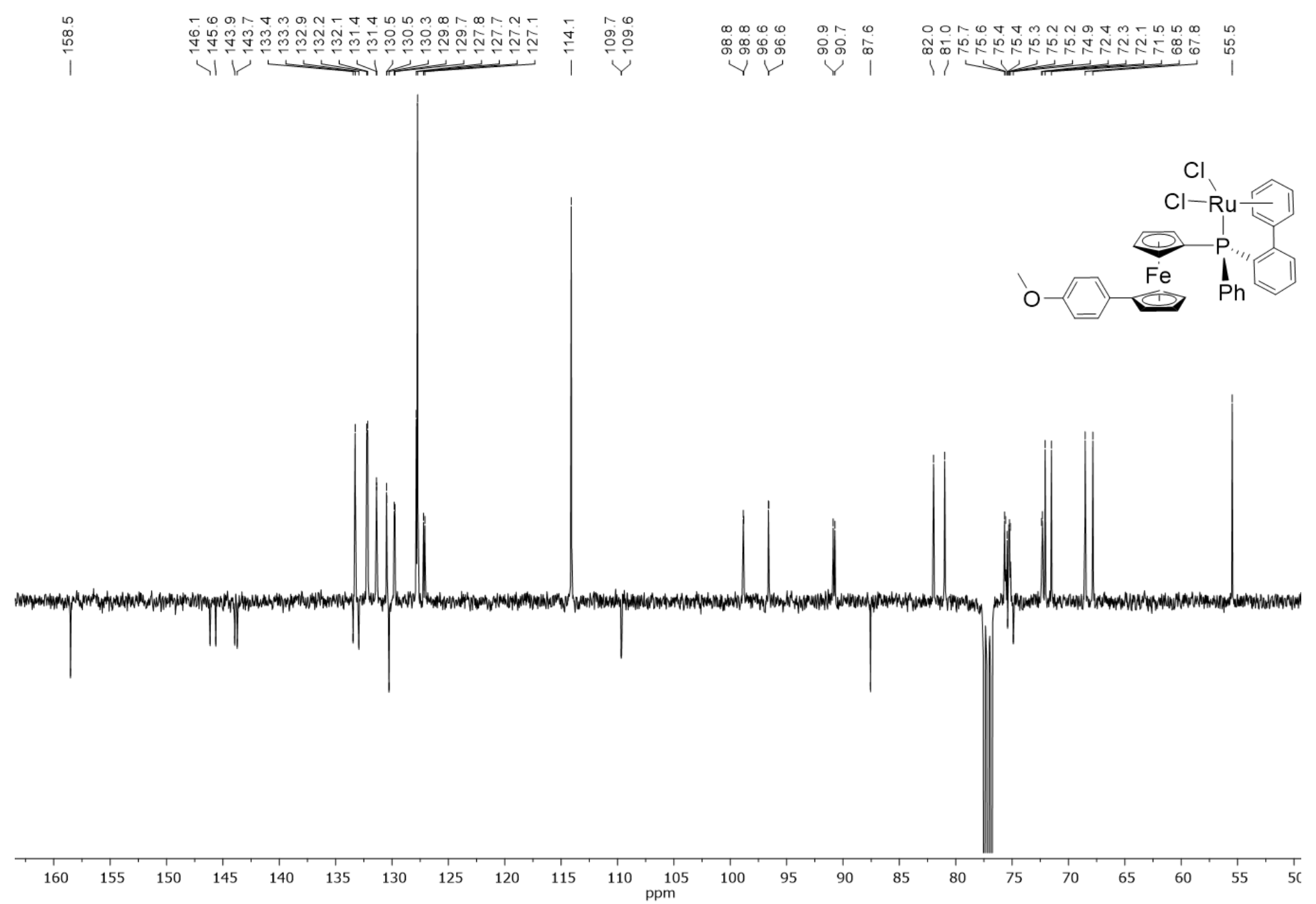

Figure S25. ${ }^{13} \mathrm{C}\left\{{ }^{1} \mathrm{H}\right\}$-NMR spectrum of $\mathbf{4 a}$. 


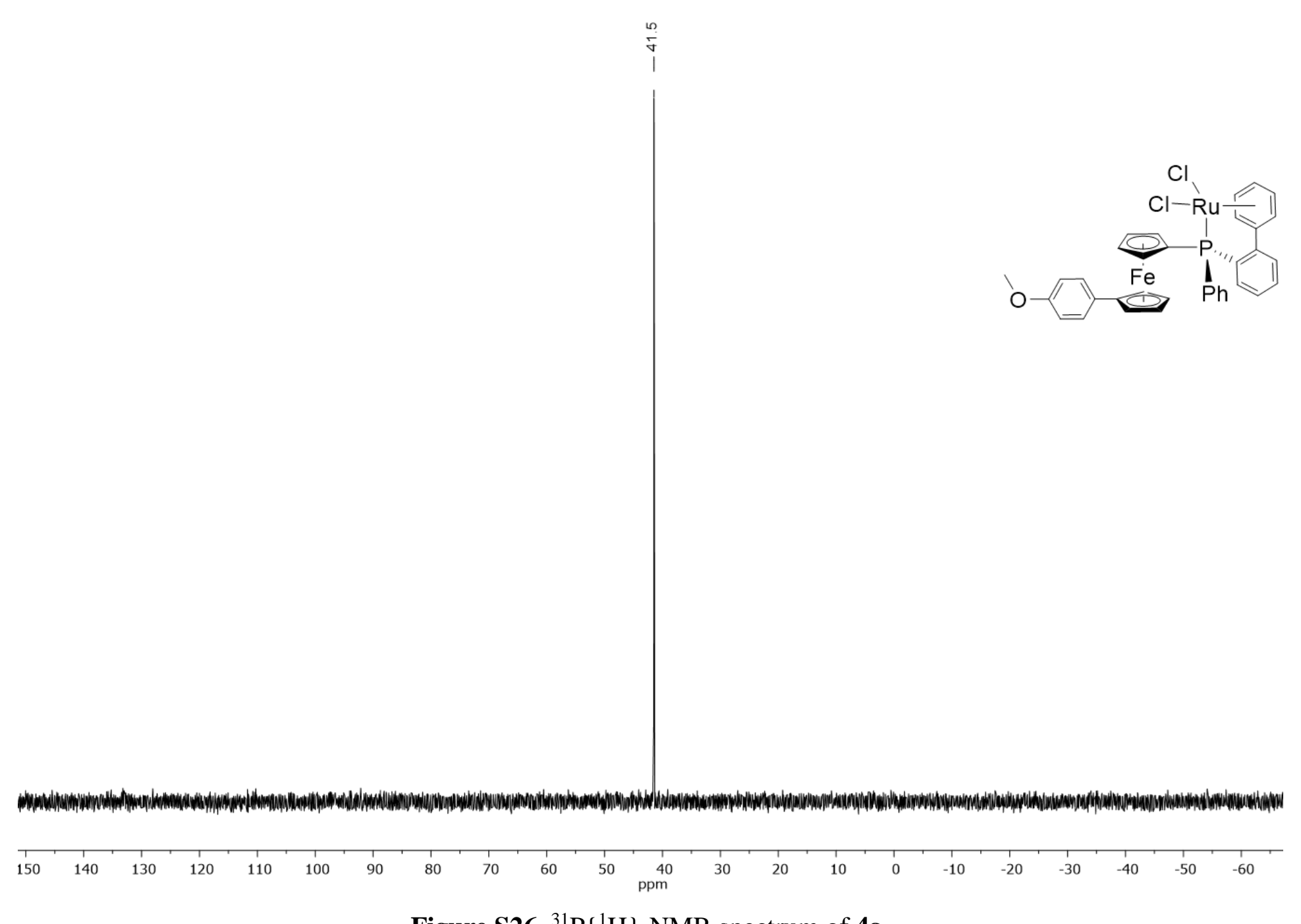

Figure S26. ${ }^{31} \mathrm{P}\left\{{ }^{1} \mathrm{H}\right\}$-NMR spectrum of 4 a. 
trans-Dichlorobis\{1-(RP)- $\kappa$ P-[2-(methoxy- $\kappa O)$ phenyl](phenyl)phosphino-1'-(4-methoxyphenyl)ferrocene\}ruthenium(II) (4b)

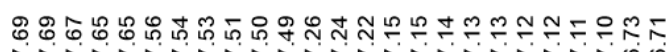

๑

管

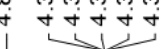

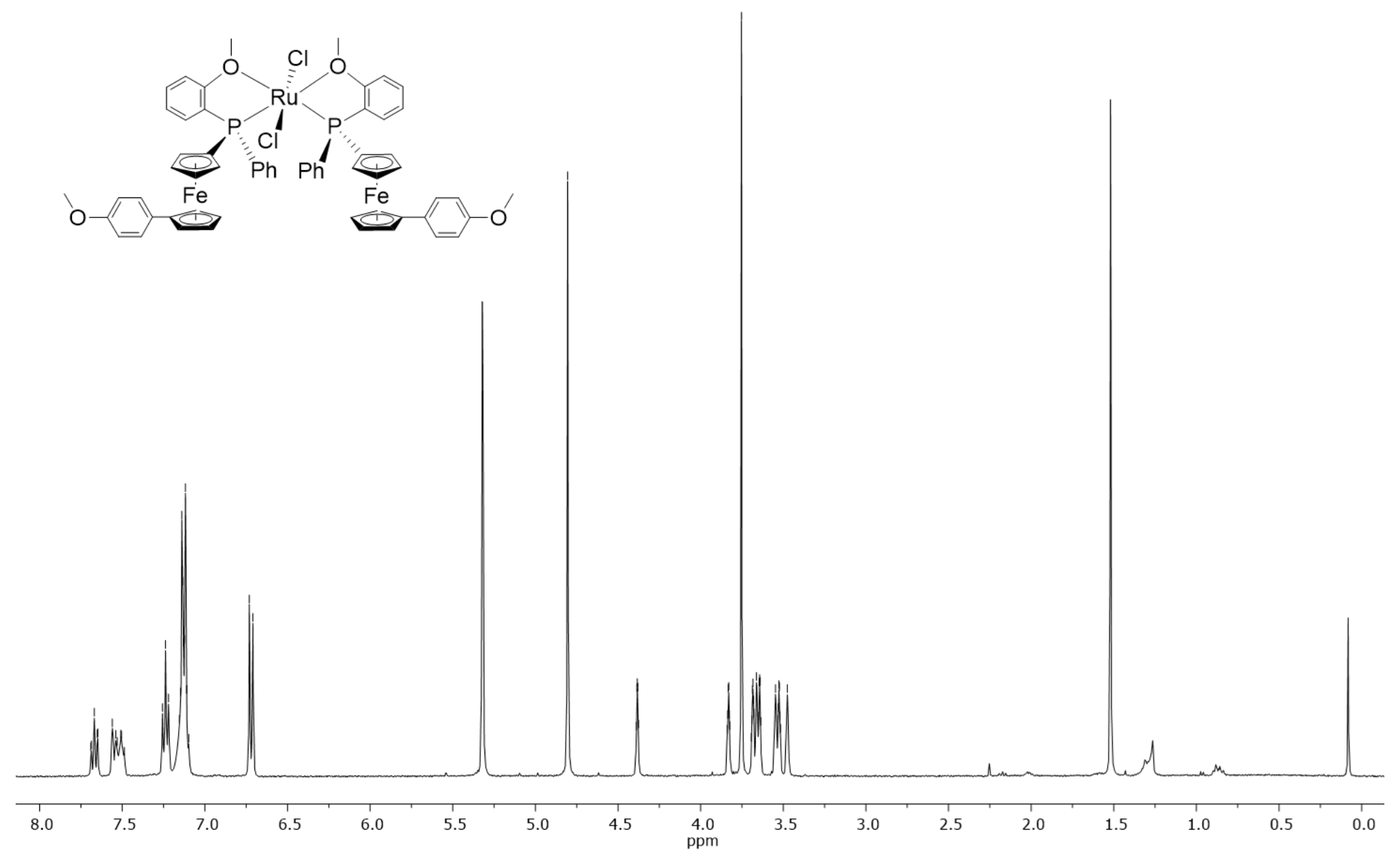

Figure S27. ${ }^{1} \mathrm{H}-\mathrm{NMR}$ spectrum of $\mathbf{4 b}$. 


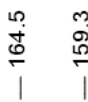

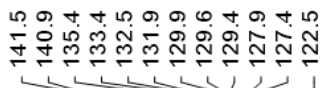
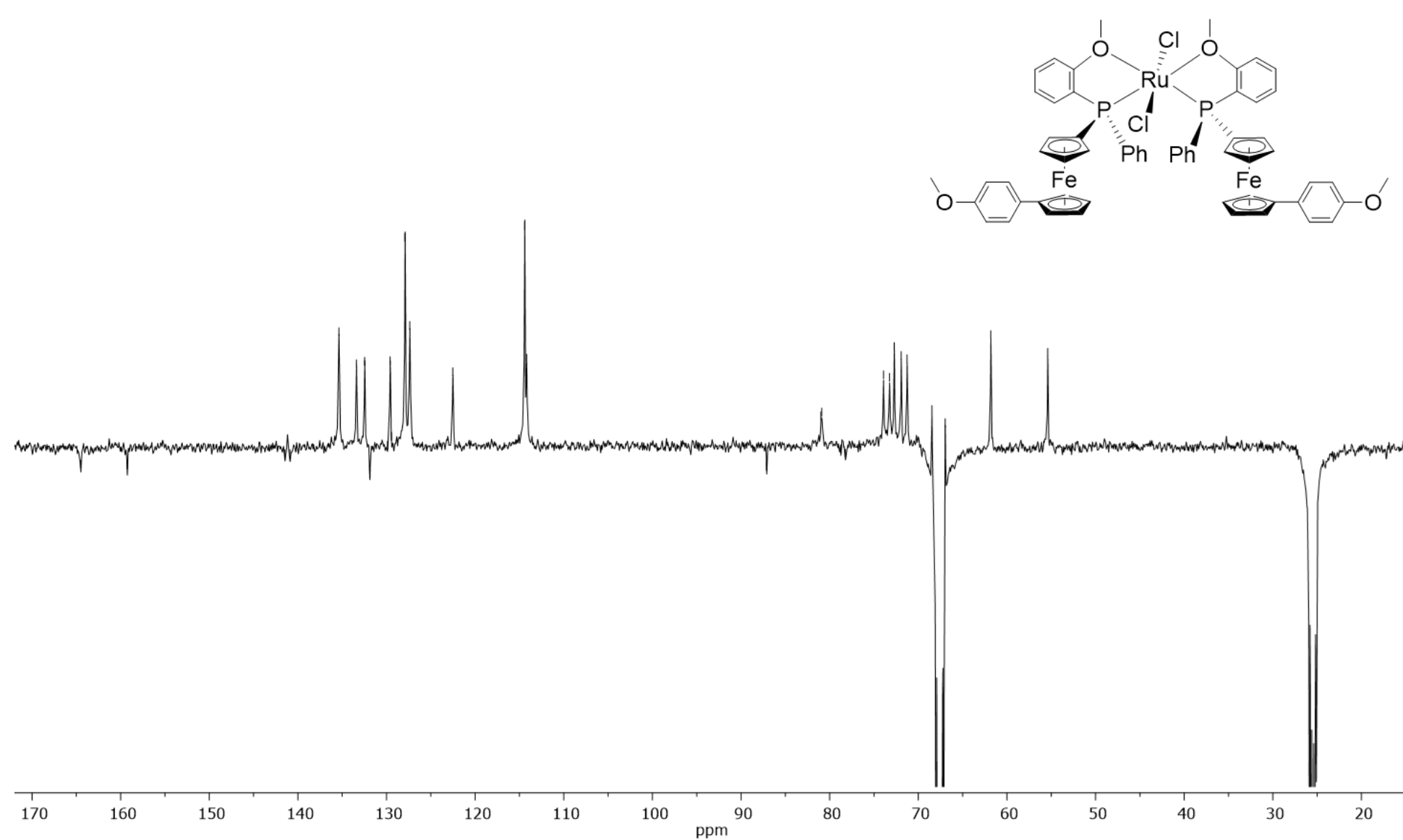

Figure S28. ${ }^{13} \mathrm{C}\left\{{ }^{1} \mathrm{H}\right\}$-NMR spectrum of $\mathbf{4 b}$. 


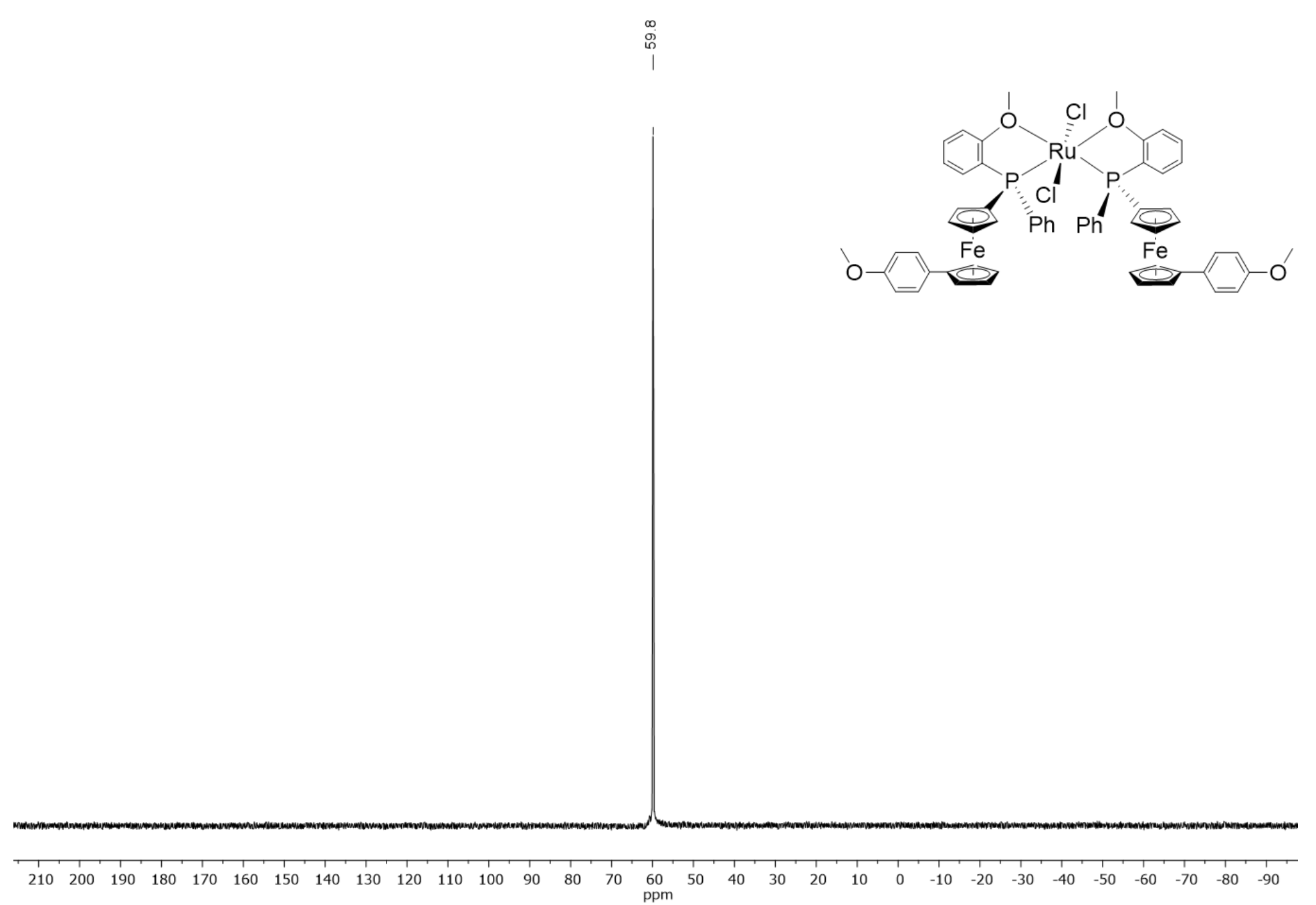

Figure S29. ${ }^{31} \mathrm{P}\left\{{ }^{1} \mathrm{H}\right\}$-NMR spectrum of $\mathbf{4 b}$. 